\title{
Investigation of Horizontal Parallax Only (HPO) light field 3D display
}

\section{Guo Song}

School of Electrical \& Electronic Engineering

A thesis submitted to the Nanyang Technological University in partial fulfillment of the requirement for the degree of Master of Engineering 


\section{Acknowledgement}

First and foremost, I would like to thank my supervisor, Prof. Sun Xiao Wei, and my co-supervisor, Prof Yuan Junsong, for their guidance and kind help during my research. I am grateful that they gave me the chance to work on the glasses-free 3D display projects. Through the research works, I enriched my knowledge in computer graphics and human-computer interaction and many other areas.

I would like to give special thanks to Dr. Xia Xinxing, as we enjoyed working together on the topic of light field display. Thanks to his ideas and suggestions, the new prototype can be finished soon.

I would like to thank Prof. Zheng Yuanjin for his support in Advanced Display Lab on my work as Project Officer. Without his help, I could never work on nice workstation to develop better programs.

I am grateful to my fellow teammates in the ADL, Mr. Zhang Lei, Mr. Zhang Xiangyu, Dr. Song Weitao, and Dr. Xia Xingxing, for the memorable discussions and the fun and laughter we have shared.

I gratefully acknowledge the funding from National Research Foundation that made my work in ADL possible.

Most importantly, my work would never be successful without the love from my family. I would like to express my deepest gratitude to my wife and my parents for their invisible help behind. 


\section{Table of contents}

Acknowledgement ........................................................................................ i

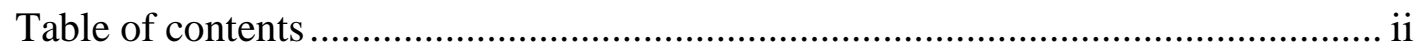

Summary

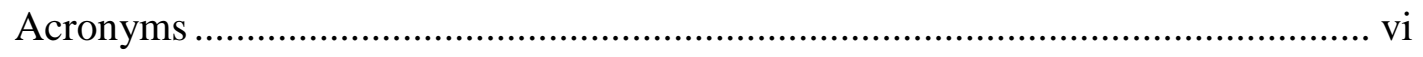

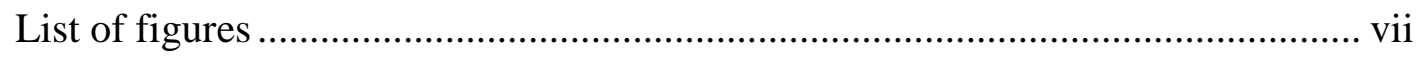

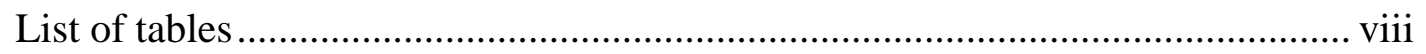

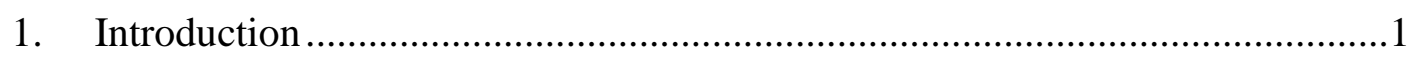

1.1. Light field display ...............................................................................

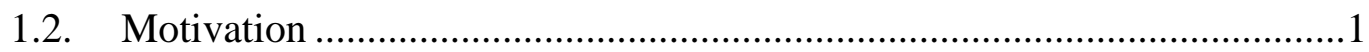

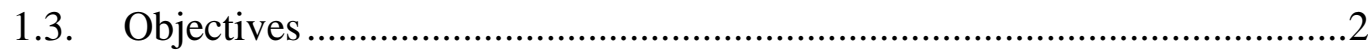

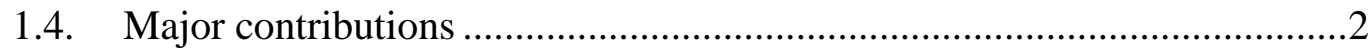

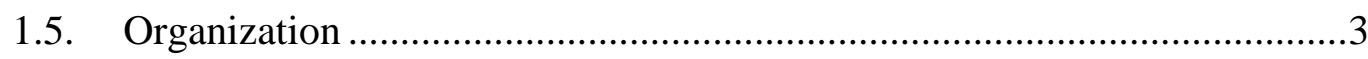

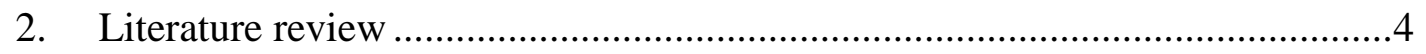

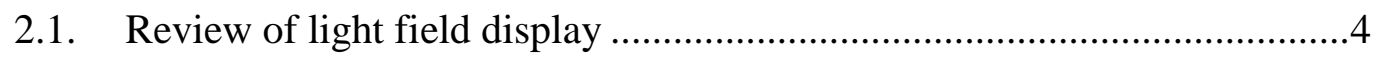

2.1.1. Light field ....................................................................................

2.1.2. Reconstruction of light field by multiple layers ....................................

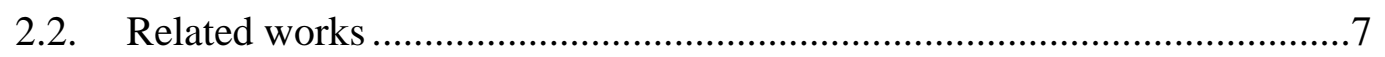




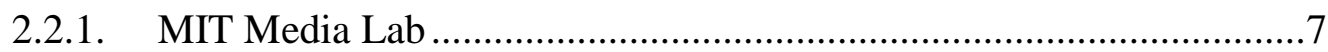

2.2.2. University of North Carolina at Chapel Hill ......................................12

2.2.3. Chinese Academy of Sciences ...........................................................13

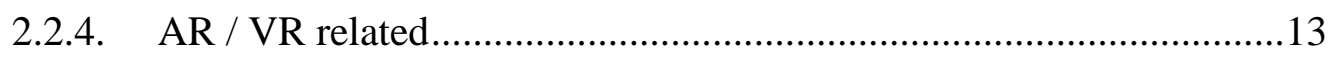

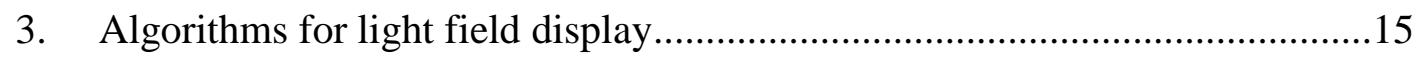

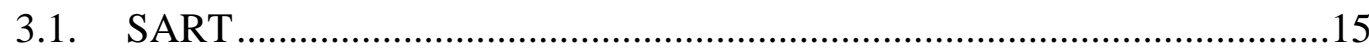

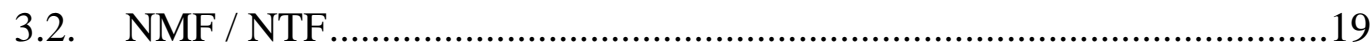

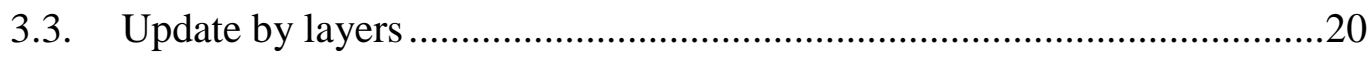

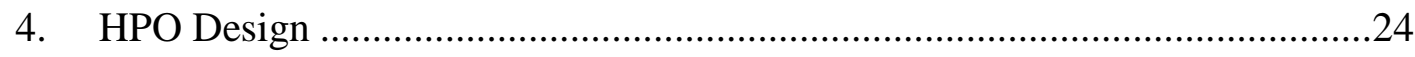

4.1. HPO Concept explanation ..........................................................................24

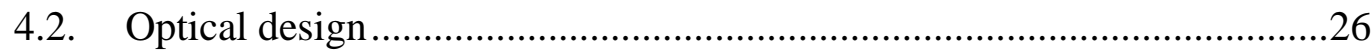

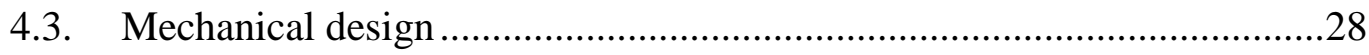

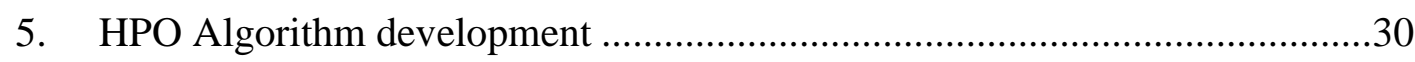

5.1. CUDA parallel computing .........................................................................

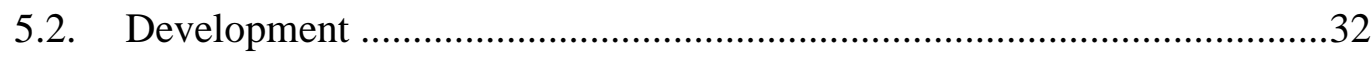

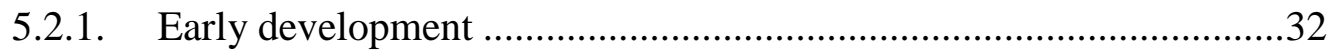

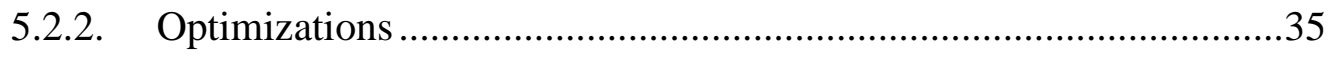

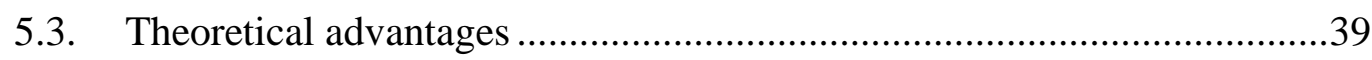

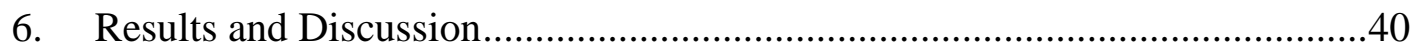

6.1. Reconstructed light field .................................................................. 
6.2. PSNR across pixel lines .......................................................................43

6.3. Computation time vs number of iterations .................................................46

6.4. Number of iterations vs Image quality ....................................................48

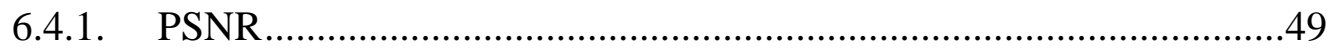

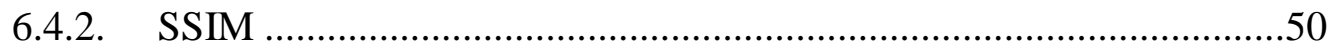

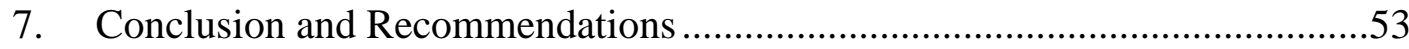

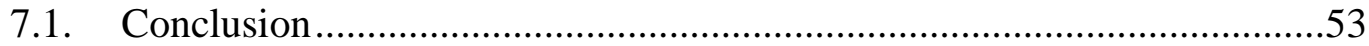

7.2. Recommendations for further research .................................................53

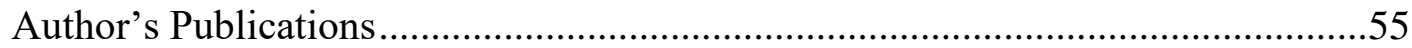

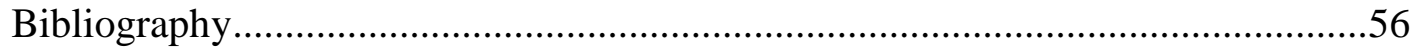




\section{Summary}

To achieve glasses-free 3D vision, light field display has been proposed and implemented for a few years, while there still exists many restrictions for such device. The most serious limit of the display is its long processing time for decomposition of input light field, as it requires computation of multiple layers to reconstruct light field at designated position. To reduce computing time while keep high image fidelity of output light field, a horizontal-only parallax method is proposed and examined in this thesis. The hardware structure, mainly backlight, has a special optical and mechanical design to support new algorithm. The input light field of new algorithm only has horizontal perspectives for efficient parallel computing. While input light field images are divided into horizontal line slices, the resultant image layer fractions are combined as full images after processing. The computation time and image quality are compared with existing works, and suggestions for further improvements are given. 


\section{Acronyms}

1D

2D

3D

LCD

NMF

NTF

HPO

CPU

GPU

PSNR

FOV

HOE

AR

VR

FBO

SSIM
One-dimensional

Two-dimensional

Three-dimensional

Liquid Crystal Display

Nonnegative Matrix Factorization

Nonnegative Tensor Factorization

Horizontal Parallax Only

Central Processing Unit

Graphics Processing Unit

Peak Signal-to-Noise Ratio

Field Of View

Holographic Optical Elements

Augmented Reality

Virtual Reality

Frame Buffer Object

Structural Similarity 


\section{List of figures}

Figure 1 Illustration of simplified light field in 2D image set form........................4

Figure 2 Simplified 1D three-layer structure. ..................................................

Figure 3 Illustration of tensor display with coordinates...................................

Figure 4 Attenuation model vs Polarization model............................................11

Figure 5 A 3D scene of multi-layer display with tracking camera. .......................12

Figure 6 SART step 1: Initialize with the target light field image set. ...................16

Figure 7 SART step 2: The emitted light field is reconstructed from pixels of layer

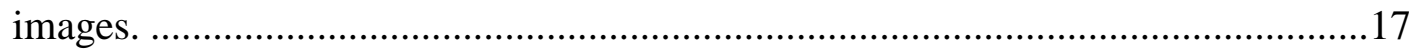

Figure 8 SART step 3: The emitted light field is compared with the target light field to find the difference.

Figure 9 SART step4: Image layers are updated based on the light field comparison result .18

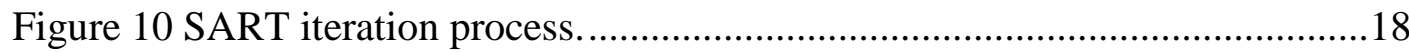

Figure 11 Illustration of update by layer method using 1D slices of three-layer

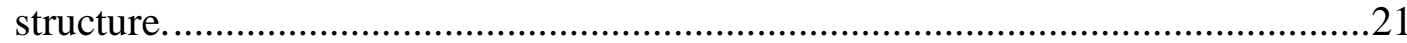

Figure 12 Two-layer HPO system configuration. ..........................................26

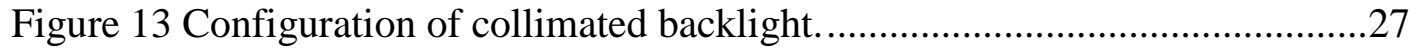

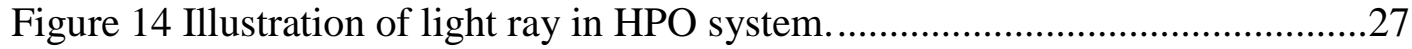

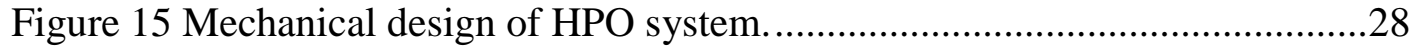

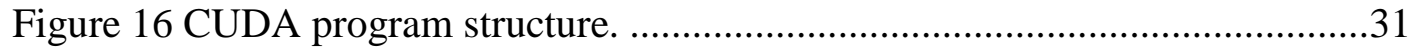

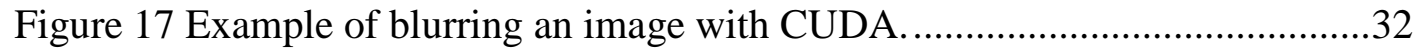

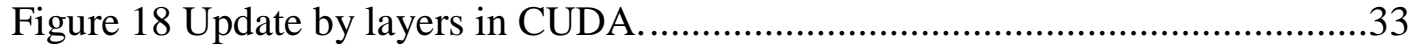

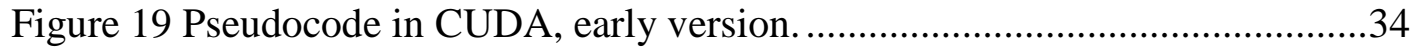

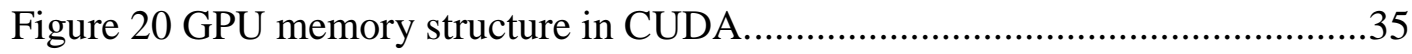


Figure 21 Pseudo code in CUDA, finalized version......................................... 38

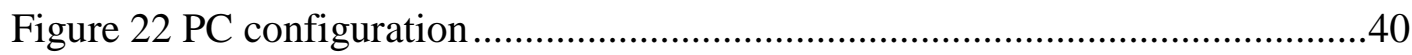

Figure 23 Parameters in both SART and HPO program...................................41

Figure 24 Original light field and reconstructed light fields of Dice with time, PSNR

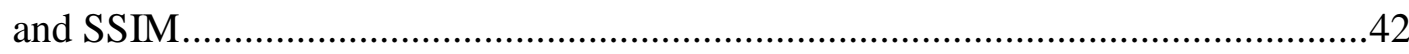

Figure 25 Original light field and reconstructed light fields of Messerschmitt with

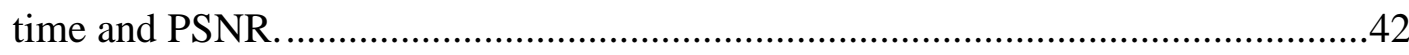

Figure 26 PSNR across lines in Dice reconstructed light fields ..........................44

Figure 27 PSNR across lines in Messerschmitt reconstructed light fields..............45 Figure 28 Computation Time vs Number of Iterations for Dice light field

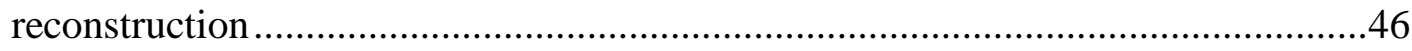
Figure 29 Computation Time vs Number of Iterations for Messerschmitt light field

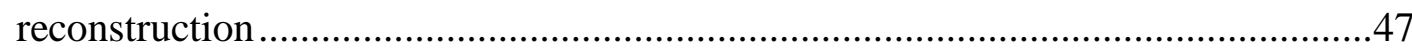

Figure 30 PSNR vs Number of Iterations for Dice light field decomposition.........49 Figure 31 PSNR vs Number of Iterations for Messerschmitt light field decomposition Figure 32 SSIM vs Number of Iterations for Dice light field decomposition .........50 Figure 33 SSIM vs Number of Iterations for Messerschmitt light field decomposition 51

\section{List of tables}

Table 1 Extra data sets tested.

.42 


\section{Introduction}

\subsection{Light field display}

Traditional 2D display is no longer a novel technology. Display manufacturers and researchers have been looking for new displays that are more attractive to consumers. A number of ideas have been proposed and implemented, and there is one kind particularly aroused people's interest, autostereoscopic display. Namely, such display can give viewers a sense of depth or 3D automatically without wearing any special eyewear. Such device is also called glasses-free 3D display. In general, autostereoscopic display includes several types, parallax barriers [1], directional backlight [2], integral imaging [3], volumetric [4], and holographic [5]. The last one, holographic display, contains detailed light field information of a scene from all angles, which involves complicated implementation and has various limitations such us high cost. A more feasible version, light field display, was then introduced to show a light field that contains a certain number of viewing angles, by using a few Liquid Crystal Display (LCD) layers and an algorithm to encode the light field inside and reconstruct it from the layers. As a result, the viewer can observe a light field in front of his eyes, which project images of different perspectives to his two eyes, and therefore forms 3D vision without help of any lenses.

\subsection{Motivation}

Current existing light field display prototypes have demonstrated great potential of such display, but also presented many drawbacks. The most fatal one that has been 
preventing it from commercialization is its tremendous amount of computation for the generation of layer images to reconstruct light field. A few methods have been applied to reduce the processing time, but it is still far from becoming a commercial product. Showing static light field is acceptable, but real-time rendering is always more attractive to customers. The calculation speed needs further improvement to achieve this, therefore an innovative design over current solutions is needed.

\subsection{Objectives}

The purpose of this thesis is to propose an HPO type of light field display and verify its improvements compared with existing prototypes.

\subsection{Major contributions}

The major contribution is to introduce and evaluate a new type of light field display. Detailed contributions are listed below:

- An HPO light field display is proposed with optical and mechanical design and special algorithm.

- The light field image set is divided into smaller fractions, namely pixel lines, for computation. Such division eliminates correlation between lines and makes parallel computing much more efficient.

- A method to accelerate layer images update by splitting input into pixel lines for calculation in Graphics Processing Unit (GPU) using CUDA programming is demonstrated. 
- In computation of layer images, a new method of memory manipulation in GPU for light field decomposition is implemented to greatly accelerate computation speed.

- Simulation result give solid proof that the proposed HPO light field display can achieve higher speed while keeping image quality, compared to prior works.

\subsection{Organization}

After this introduction part, chapter 2 gives a review of related works on the light field display, including different prototypes developed so far and various algorithms used. Chapter 3 goes deeper on the algorithms for decomposing light field, and discusses potential improvements

Chapter 4 describes the design of the HPO, where both optical and mechanical details are given. In chapter 5, the development of algorithm used for HPO is given, with how the optimizations are implemented.

Chapter 6 gives information on assessments conducted using the new algorithm of HPO. The technical specifications are given, and the simulation results are compared with existing methods.

In the last chapters, a conclusion of this thesis is drawn first, followed by what can be done for future researches. 


\section{Literature review}

\subsection{Review of light field display}

\subsubsection{Light field}

To understand what is a light field display, the concept of light field must be explained first. A light field is an interpretation of light as a field that contains information of light in all direction through every point in a space. However, it is too complicated to use any existing device to fully record and reconstruct such great amount of information. To make it more feasible for recording and reconstruction, we can limit the number of perspectives and image a light field to be set of 2D images [6], whereas each of them shows a perspective of a 3D scene or object, as shown in a simplified illustration below.

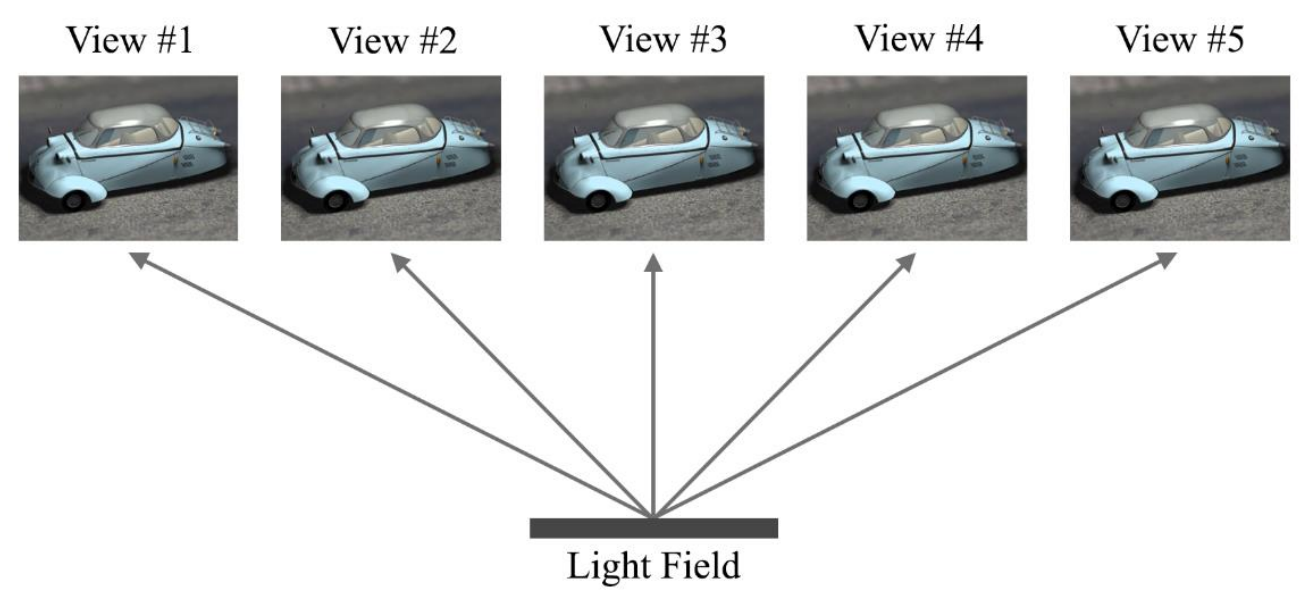

Figure 1 Illustration of simplified light field in 2D image set form. Five views from five different perspectives can be observed at five different viewing angles. The distances, angles, and length of arrows are drawn for demonstration only, not in real scale. Light field image set of Messerschmitt is obtained from MIT Media Lab light field archive [7]. 
By viewing the light field from different angles, images of different perspectives could be seen. Consequently, with proper gap between views, viewers can see two different perspectives in two eyes, and therefore see 3D from the light field.

\subsubsection{Reconstruction of light field by multiple layers}

With an established light field source, which is usually in the form of a set of images of a scene or object captured from a series of viewing angles, an output device is needed to reconstruct the light field to viewers. In order to reproduce a light field showing original different perspectives, MIT Media Lab built a multi-layer display. Although they are not the first one to propose multi-layer solution, their solution have fewer artifacts and a larger depth of field [8].

How can a light field with many different views be reconstructed with just a few layers of screens? An illustration of multi-layer display can explain this as shown below. 1D slices of a three-layer display is drawn for simplicity.

(a)

$$
\begin{aligned}
& I_{f}(25) \times I_{m}(25) \times I_{r}(26)=L_{1} \\
& I_{f}(25) \times I_{m}(24) \times I_{r}(23)=L_{2}
\end{aligned}
$$

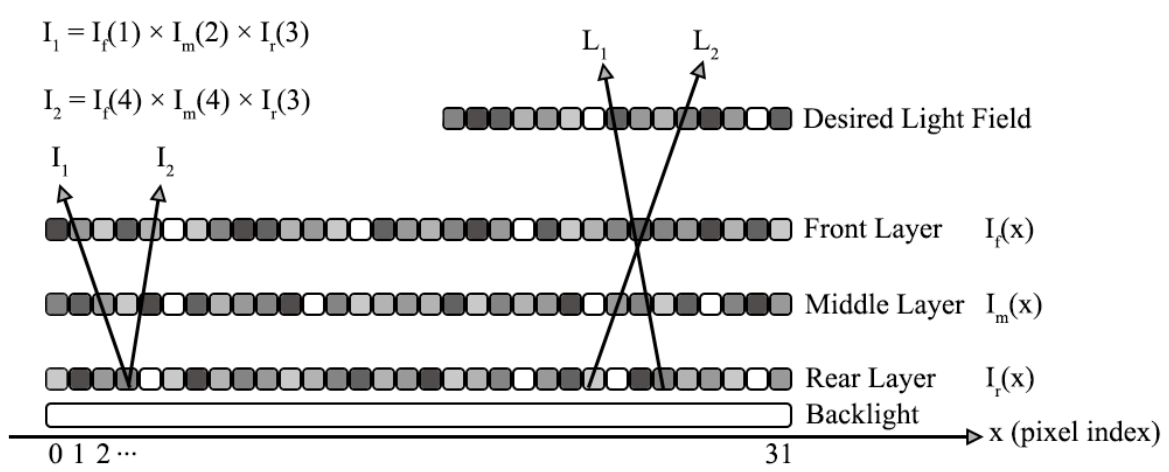

Figure 2 Simplified 1D three-layer structure. (a) Reuse of single pixel on layers. (b) generating multiple perspectives of light field at desired position. Grayscale is used to 
represent intensity of pixels, which can be interpreted as any color channel. I() stands for the intensity. " $x$ ” denotes multiplication here (could be addition in other designs).

On the left part (a) of the image, two rays are traced from one same pixel on rear layer. By tracing two rays of different directions, two intensities could be observed by associating pixels the ray travels through (denoted by " $x$ " in the image). This implies that pixels on multiple layers can be reused for different perspectives.

As a result, right part (b) of the image can be achieved. To generate a target perspective at desired light field position, rays could be traced back to the multiple layers. The intensity of the rays can be calculated by associating pixels along the ray. Since all pixels on the layers can be reused many times, through calculation the designated light field with many perspectives can be reconstructed.

As shown in (b), two target rays, $\mathrm{L}_{1} \& \mathrm{~L}_{2}$, are from two different perspectives of two light field positions, both need pixel 25 on front layer, and they also travel through pixels on other planes. Then the intensity of pixel 25 on front layer can be carefully chosen to satisfy both equations shown in the image. In this way, pixel 25 is reused by two views from two light field positions.

This rule can be expanded to more views, like 7 in horizontal direction and 7 in vertical direction, where all pixels on all the layers are reused by these 49 views. However, with more views, the equations to achieve target light field intensity values are more difficult to satisfy. Namely, the reconstructed light field intensity values can only be close to the target value, which depends on algorithm. Therefore, the key part of the multi-layer light field display is the calculation of layer pixel values to reproduce target light field. Several works on this will be introduced in next chapter. 


\subsection{Related works}

\subsubsection{MIT Media Lab}

\subsubsection{Early works}

Initially the MIT Media Lab start from dual-layer LCD structure. By treating the layers as content-adaptive barriers, or spatial light modulators, the backlight is modulated to recreate a light field. Their prototype consists of backlight, rear layer, spacer, front layer, and a polarizer from back to front, and the scene they use is computer generated [9].

To decompose the target light field, they introduce Nonnegative Matrix Factorization (NMF) method, which will be explained later. However, due to both hardware and software tool used for computation, it takes eight minutes to render one single frame, which requires at least 50 iterations to achieve a good Peak Signal-to-Noise Ratio (PSNR) of $28 \mathrm{~dB}$ [9]. Other than computation speed limitation, only simple 3D objects are demonstrated.

In a later research, they use light-attenuating material, clear acrylic sheets with printed image patterns, to form the layers and prove the feasibility of multi-layer displays based on tomographic principles [8].

Using this prototype, they prove that the tomographic design works and multi-layer displays have equivalent or better abilities than volumetric and autostereoscopic displays. Unlike volumetric displays, such display not only can show inward depth cues, but also produce perceptual depth out of the display. While parallax barriers and integral imaging displays lose resolution when reproducing $3 \mathrm{D}$, this display can 
show high-resolution images. However, these benefits do not come with no cost. An increasing amount of computation is needed. Moreover, this prototype can only show printed static image [8].

\subsubsection{Tensor Display}

By changing the media for light field reconstruction from printed acrylic sheets to LCD screens, MIT Media Lab introduce a new multi-layer light field display, tensor display.

Basically, they use three LCD screens to emit a light field in front of viewers [10]. To represent a light field formed by a tensor display with $\mathrm{N}$ layers and $\mathrm{M}$ frames, a sparse set of non-zero elements limited to a plane with an $\mathrm{N}^{\text {th }}$-order, rank-M tensor is used for analysis [10]. Take three-layer display for example, a simplified illustration of part of the display is shown below.

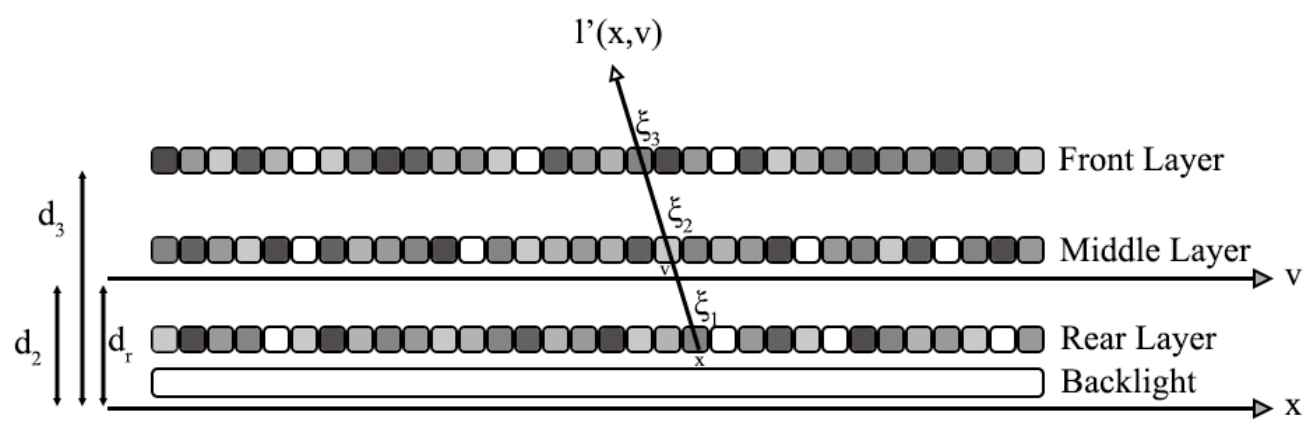

Figure 3 Illustration of tensor display with coordinates. From top to bottom: front layer, middle layer, and rear layer. Three light-attenuating LCD layers are illuminated by a omnidirectional backlight from the back of rear display.

As illustrated in the image, 1D slices of three layers are producing a 2D light field. While $l(x, v)$ denotes the target light field, $l^{\prime}(x, v)$ stands for the emitted light field by the layers. The whole reconstructed light field of $\mathrm{N}$ layers can be expressed as: 


$$
l^{\prime}(x, v ; N)=\prod_{n=1}^{N} f^{(n)}\left(x+\left(\frac{d_{n}}{d_{r}}\right) v\right),
$$

where $x$ and $v$ are the coordinates on rear layer and middle layer respectively (which determines direction of rays), and $f^{(n)}\left(\xi_{n}\right)$ is the transmittance at point $\xi_{n}$ on layer $n$, while the $\mathrm{n}^{\text {th }}$ layer is $d_{n}$ away from the rear layer $\left(d_{r}\right.$ is equivalent with $\left.d_{2}\right)$. Since this is a three-layer structure, suppose the transmittance of the three layers, from rear to front, are $f\left(\xi_{1}\right), g\left(\xi_{2}\right)$, and $h\left(\xi_{3}\right)$ respectively, then the emitted light field of this three-layer display can be represented as:

$$
l^{\prime}(x, v)=f\left(\xi_{1}\right) g\left(\xi_{2}\right) h\left(\xi_{3}\right), \text { where } \xi_{n}=x+\left(\frac{d_{n}}{d_{r}}\right) v
$$

Therefore, a restriction of the function below can parameterize the emitted light field

$$
t\left(\xi_{1}, \xi_{2}, \xi_{3}\right)=f\left(\xi_{1}\right) g\left(\xi_{2}\right) h\left(\xi_{3}\right)
$$

which is defined in the $3 \mathrm{D}$ Euclidean space $\mathbb{R}^{3}$ spanned by $\left\{\xi_{1}, \xi_{2}, \xi_{3}\right\}$, to the $2 \mathrm{D}$ subspace formed by $\alpha \xi_{1}+\beta \xi_{2}+\gamma \xi_{3}=0$, with

$$
\alpha=d_{3}-d_{2}, \beta=d_{1}-d_{3}, \gamma=d_{2}-d_{1}
$$

Each light ray $(x, v)$ in the image above can be represented by a series of intersection $\left\{\xi_{1}, \xi_{2}, \xi_{3}, \ldots, \xi_{n}\right\}$. For the three-layer LCD display, where pixels are discrete, the intensities of the reconstructed light field $l^{\prime}\left\{\xi_{1}, \xi_{2}, \xi_{3}\right\}$ can be represented by $f_{i} g_{j} h_{k}$ approximately, where $\{\mathrm{i}, \mathrm{j}, \mathrm{k}\}$ stands for the closest integer pixel index of the intersections. Now the equation 3 can be replaced with a $3^{\text {rd }}$-order, rank-1 tensor $\mathrm{T}$ in discrete coordinates,

$$
T=f \circ g \circ h, \text { such that } t_{i j k}=f_{i} g_{j} h_{k}
$$


where $\circ$ is the vector outer product. Note that only part of the tensor elements $t_{i j k}$ are valid light field rays, as limit has been stated above. To make such limitation even clearer, a binary-valued tensor $\mathrm{W}$ is introduced such that the emitted light field is given by:

$$
L^{\prime}=W \circledast T, \text { for } w_{i j k}=\left\{\begin{array}{c}
1 \text { if }\{i, j, k\} \text { gives a light field ray } \\
0 \text { otherwise }
\end{array}\right.
$$

where $\circledast$ is the Hadamard (elementwise) product.

With this tensor representation of the emitted light field, the error between emitted light field and the target light field can be given, and such error should be minimized. The objective function can be expressed as:

$$
\arg \min _{F, G, H}\|L-W \circledast \llbracket F, G, H \rrbracket\|^{2}, \text { for } 0 \leq F, G, H \leq 1
$$

where $L$ is the target light field tensor, F, G, and $\mathrm{H}$ denotes three layer tensors.

Following the equations above, if the objective function is solved, the error between reconstructed light field and the desired light field can be diminished [10]. To solve this, Nonnegative Tensor Factorization (NTF) was used, which is a special form of NMF mentioned previously and will be covered later.

Consequently, the decomposition of the target light field into multiple LCD layers has been proved successful, as the resultant perspective images have acceptable PSNR [10]. Nevertheless, several improvements are needed, such as brightness, image fidelity, and computation speed. 


\subsubsection{Polarization Field Display}

To mitigate the dim output light field images problem, MIT Media Lab proposed a different optical design [11].

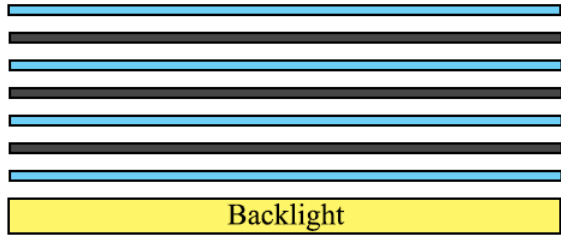

Attenuation model

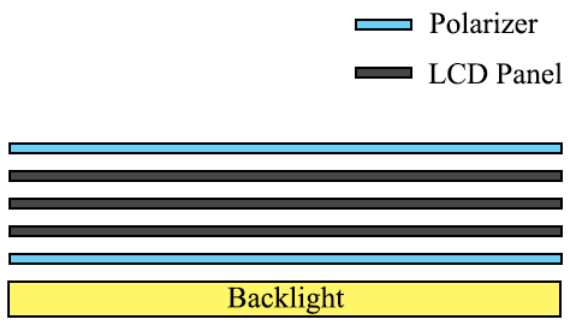

Polarization model

Figure 4 Attenuation model vs Polarization model.

Compared with the attenuation-based design, the polarization model requires more modification on both hardware and software algorithm. As polarizers cannot transmit $100 \%$ of light, the resultant image of the polarization design with fewer polarizers is brighter.

However, the cost of such design is the polarization calculation. The output intensity of each LCD layer must be converted by Malus' law:

$$
I=I_{0} \sin ^{2}(\theta)
$$

where $I_{0}$ is the initial intensity, and $\theta$ is the polarization angle of the layer.

The objective function and error minimization process is similar to the tensor prototype, but a different algorithm, simultaneous algebraic reconstruction technique (SART), is introduced to solve the problem [11]. This method is implemented on GPU and proved to be efficient, and details will be given in later part. However, to 
achieve real-time rendering of light fields, the computation speed still needs improvement.

\subsubsection{University of North Carolina at Chapel Hill}

In collaboration with MIT Media Lab, Andrew Maimone from University of North Carolina goes further on the multi-layer light field display [12]. The previous prototypes have limited Field Of View (FOV), usually 10 degrees in both horizontal and vertical direction. To overcome this disadvantage, a tracking device, Microsoft Kinect sensor is added to locate viewer's position and adapt content accordingly. For example, when user can only see an object's left side at one position, he can move towards right to see the right side. Actually, the FOV of each static computed light field itself does not change, but the content and the alignment between layers adjust programmatically based on tracking status, which therefore the perceived FOV increases.

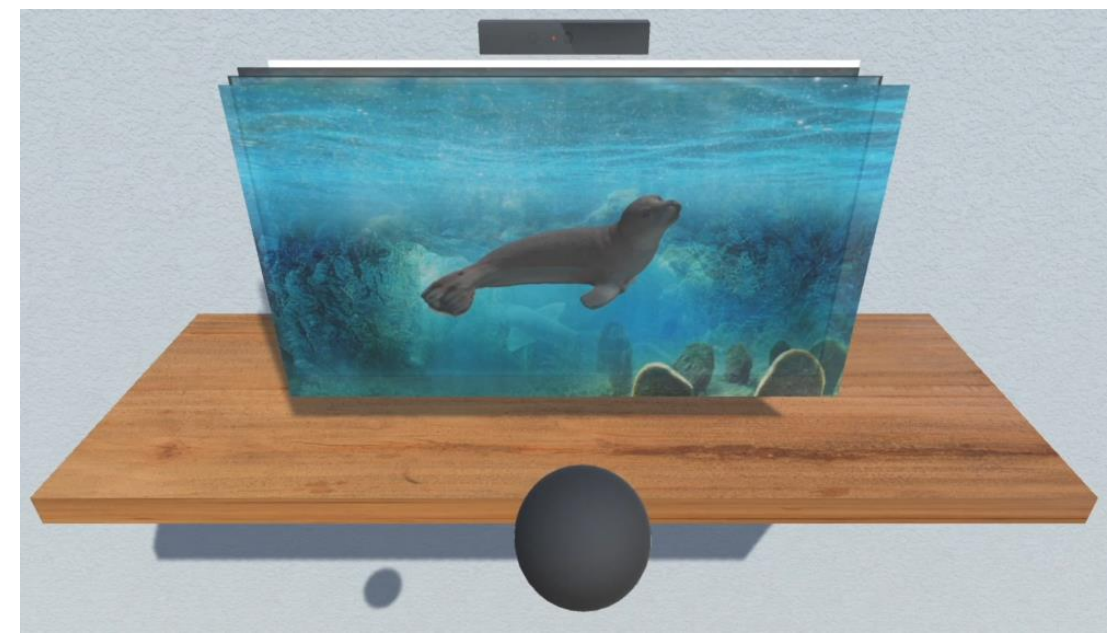

Figure 5 A 3D scene of multi-layer display with tracking camera. The black ball represents user's head, and the black bar above the layers is the Intel RealSense camera. All models and textures are self-made in Unity $3 D$. 
The FOV is greatly enlarged by this method, but such method is applicable to single user only [12].

\subsubsection{Chinese Academy of Sciences}

Xuan Cao and his colleagues from Chinese Academy of Sciences propose a different algorithm of forming the target light field by LCD layers [13]. The goal is still minimizing the difference between the emitted light field and the target light field, but Cao's algorithm focuses on updating one layer at a time. While SART does not seem to have any physical meaning in light field decomposition (since it is a graphical solver), NMF / NTF involves calculation of massive sparse matrix. The layers are updated one by one and only non-zero light rays are considered, thus less calculation is required. When one layer is being updated, other layers are kept fixed, and all valid rays of each pixel are evaluated to choose the best pixel values within this update iteration. Details of the algorithm will be given later.

In addition, the method is suitable for parallel computing. Cao implemented the algorithm in CUDA (Parallel programming tool, to be covered later), which took less than 1 second for single image, but it is still at similar level with the SART method and needs optimization.

\subsubsection{AR / VR related}

As mentioned in the beginning of this thesis, light field displays are very similar to holographic displays. Seungjae Lee and his team from Seoul National University apply Holographic Optical Elements (HOEs) to make additive light field display. Just like a holographic display, two projectors launch beams to two separate layers, 
which diffuses the projections and functions as additive layers, where additive light fields are generated independently on each layer then merges as one [14].

The intensity of the merged wave can be expressed as:

$$
I_{\text {total }}=I_{1}+I_{2}
$$

Unlike the multiplication in equation (1), addition is used in this design and provides a more straightforward calculation.

This novel design provides a see-through kind of multi-layer display, which has great potential in Augmented Reality (AR), but the optical design is too complicated to become a practical solution in near future.

In another research by Stanford University, the multi-layer display structure is applied on a Virtual Reality (VR) system, to allow users focus at different positions. Unlike current popular VR headsets, viewers are not forced to focus at a fixed distance, therefore eyes become less stressful [15]. 


\section{Algorithms for light field display}

To compute layer images for light field display, several algorithms can be used, and they all base on similar concept. This chapter introduces how these algorithms solve the problem.

As mentioned related works, the key to decompose target light field into layer images is to minimize the error between emitted light field and the target light field, as described by rule:

$$
\arg \min \left\|L-L^{\prime}\right\|^{2}, \text { for } L^{\prime} \text { within appropriate range }
$$

where $L$ and $L^{\prime}$ denotes target light field and reconstructed light field respectively. The range of $L^{\prime}$ may be different in various designs, most of which are usually $[0,1]$. This equation has various interpretation in different algorithms. One may adopt a graphic approach using computer light ray simulation, while another one may achieve it through pure mathematical matrix calculation. Moreover, it is also possible to apply a mathematical approach with light ray calculation considered.

Algorithms with these different interpretations are discussed in this chapter.

\subsection{SART}

The SART method is implemented in OpenGL, a computer graphics software [8]. In OpenGL, simulation of both light field and layers can be achieved. Although it is not possible to directly display a light field emitting perspectives to different angles simultaneously, it is feasible to show one perspective at a time. In this way, pixels of different views become correlated. The layers can be simulated by quads with 
textures located at proper positions. Shader, a tool to paint pixels based on their coordinates in $3 \mathrm{D}$ space, can relate the pixels on different layers to generate an emitted light field, one perspective at a time. A convenient tool to temporarily store the reconstructed light field and layer images data is Frame Buffer Object (FBO). The full process of SART computation of three layer is illustrated as shown below.

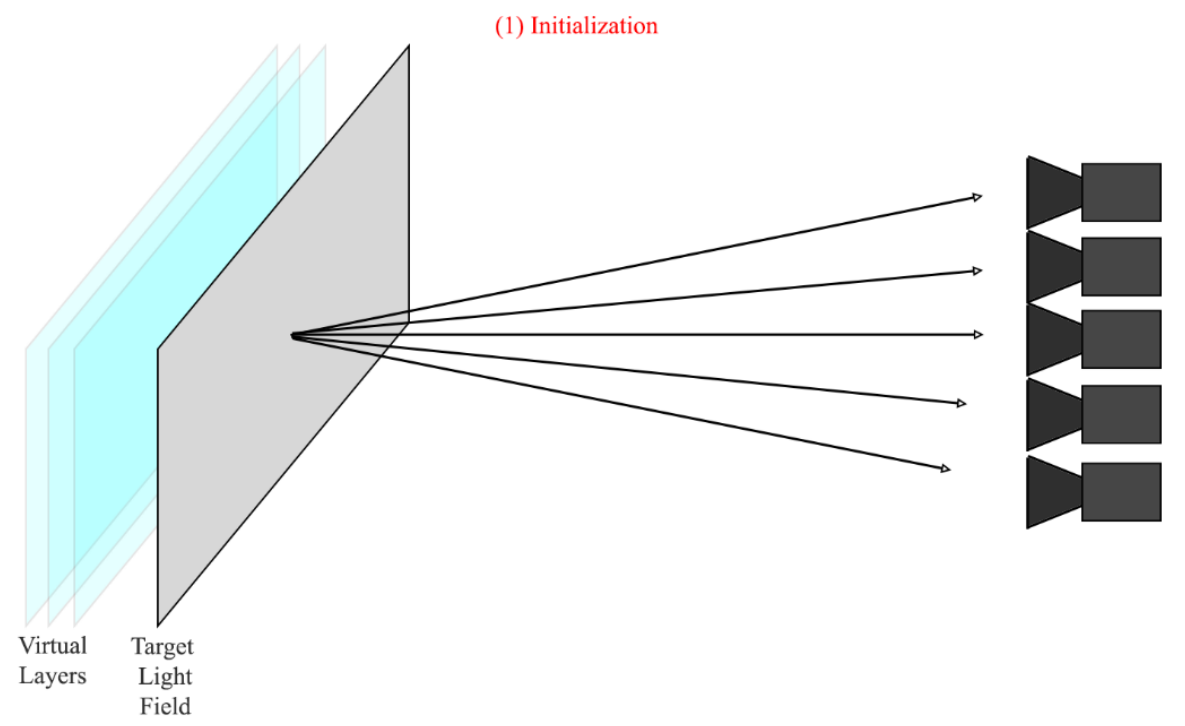

Figure 6 SART step 1: Initialize with the target light field image set. By placing the virtual cameras at designated viewing angles and load target light field images as textures for the respective perspective, the whole light field can be stored in FBOs for later steps. 


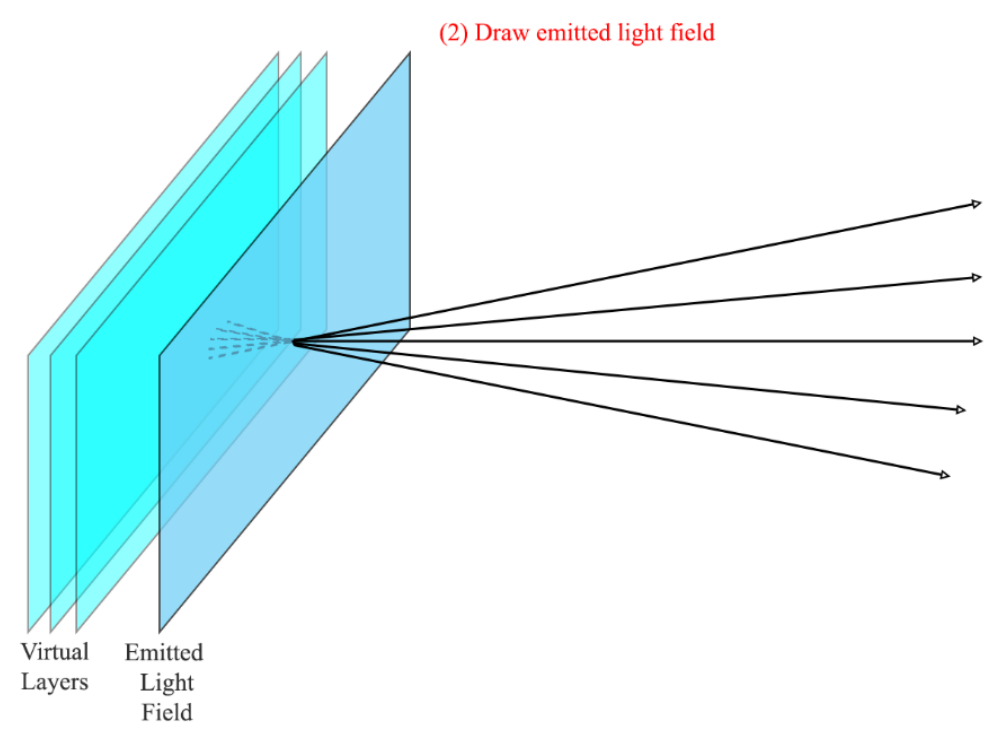

Figure 7 SART step 2: The emitted light field is reconstructed from pixels of layer images. Three virtual layers are initialized with random values at first and reads data from FBOs in later iterations, then projections from layers to target light field position at all designated viewing angles are done to obtain the reconstructed light field. Shader can ensure that the pixel values on same light ray are multiplied, with transmittance considered. Parallel projection is used to avoid distortion, since it is not concerned in the design. Resultant reconstructed light field is stored in FBOs

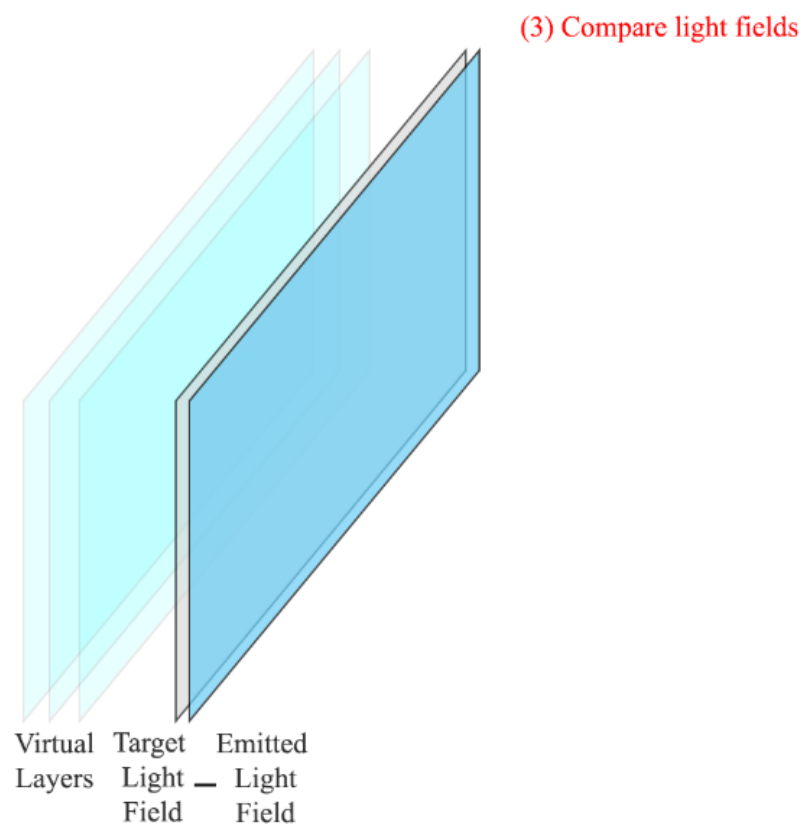

Figure 8 SART step 3: The emitted light field is compared with the target light field to find the difference. The difference is calculated in shader, and result is updated to reconstructed light field's FBOs. 
(4) Update Layers

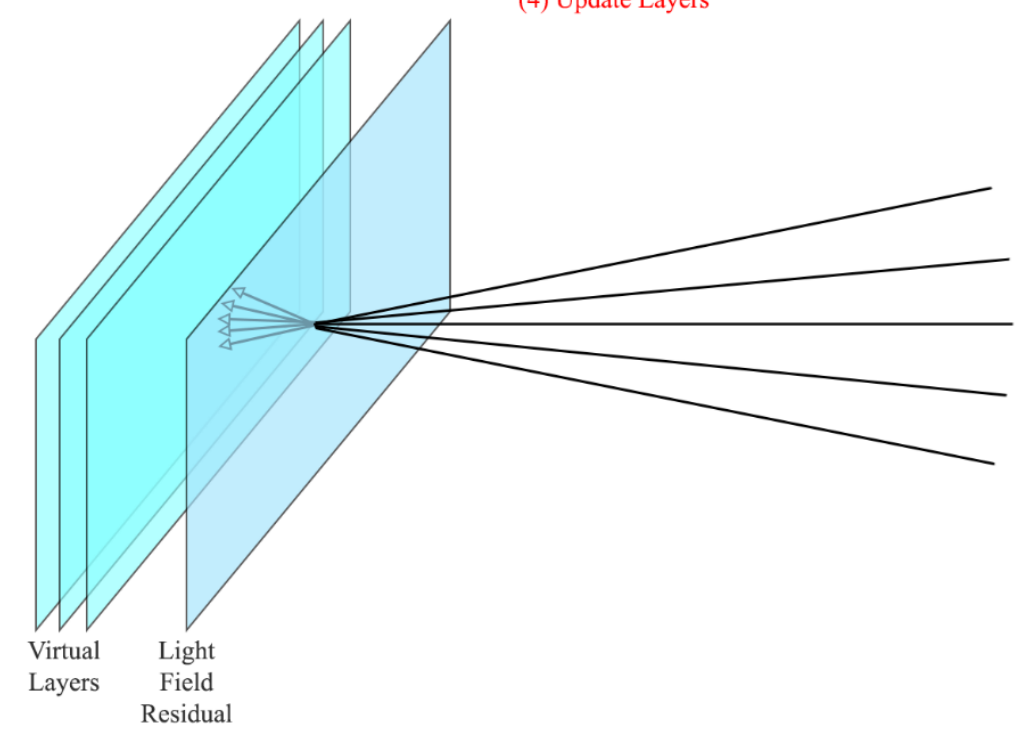

Figure 9 SART step4: Image layers are updated based on the light field comparison result. Layers are updated one by one using the residual calculated in step 3. Results are stored in image layers' $\mathrm{FBOs.}$

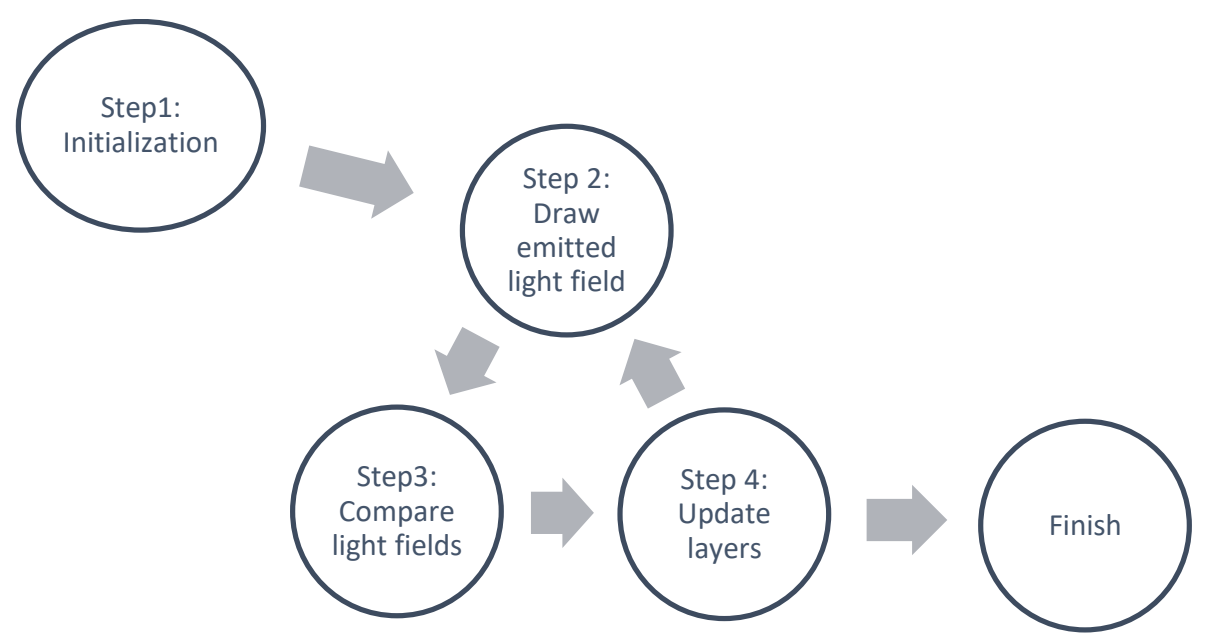

Figure 10 SART iteration process. When maximum number of iterations is achieved, the program stops and output result of layer images and reconstructed light fields.

This SART method runs on GPU, and it is based on OpenGL and Cg. The efficiency is considerable, in terms of computation speed and image quality [8], but there exists one key drawback regarding memory access. In each step mentioned above, the textures must be loaded from FBOs, which are temporarily saved in GPU's memory, 
and the results are written back to FBOs when one step finishes. In GPU, there is a faster memory type, called shared memory in CUDA (to be covered later), but not directly accessible for OpenGL. Therefore, utilizing faster memories can make calculation even faster, which is the main goal of this thesis.

We managed to reproduce and modify this algorithm to go further from MIT Media Lab's research. A direct comparison was made between this method and the proposed HPO method, and details will be given in results part.

\subsection{NMF / NTF}

To decompose the target light field into several image layers, a mathematical method that involves matrix calculation can be used.

Suppose there is a matrix $\mathrm{V}$ of $\mathrm{m} \times \mathrm{n}$ elements of nonnegative values only, it is possible to find two matrices $\mathrm{W}$ and $\mathrm{H}$, also with nonnegative elements only, such that they can satisfy the relationship:

$$
V \approx W H
$$

where $\mathrm{W}$ is a $\mathrm{m} \times \mathrm{r}$ matrix and $\mathrm{H}$ is a $\mathrm{r} \times \mathrm{n}$ matrix, and $\mathrm{r}<\min (\mathrm{m}, \mathrm{n})$ [16].

The process of finding such approximation of one single large matrix by two smaller ones is called NMF.

To minimize the error between $\mathrm{V}$ and $\mathrm{WH}$, a method similar to the SART above is used. W and $\mathrm{H}$ are updated one at a time, just like the layers that are updated in SART step 4. The update rule of NMF can be given as follows:

$$
W \rightarrow W \circledast \frac{V \circledast H^{T}}{(W H) H^{T}}, \quad H \rightarrow H \circledast \frac{W^{T} \circledast V}{W^{T}(W H)}
$$


where $\circledast$ denotes Hadamard (elementwise) product.

If we transform target light field into a sparse nonnegative matrix replace $\mathrm{V}$ with it, the resultant $\mathrm{W}$ and $\mathrm{H}$ will be the layers' pixel values [10].

The method can also be applied to more layers, like 3,4 , or even more, while the name of the process is called NTF. Of course, having more layers leads to heavier computation load. The initial implementation on Central Processing Unit (CPU) was extremely slow, i.e. 10 seconds per iteration for $5 \times 3$ views of $840 \times 525$ images and at least 50 iterations are needed for good image quality [9]. Improvements in later researches in terms of computation speed are clearly specified. It took a CUDA program 1 second to run hundreds of iterations to finish optimization in Chen's work [17]. In Maimone's result, one iteration costed 4.44 milliseconds on average and optimization need a few iterations, but the result is based on consecutive rendering between visually similar frames, which means the time taken from initialization of layers is not mentioned, and that could possibly take much more time (hundreds of milliseconds, based on experience that the initiation process costs large amount of time). None of these works give detailed information regarding computation speed, thus only indirect comparison can be made.

\subsection{Update by layers}

Unlike visual solution, SART, and mathematical calculation based solution, NMF / NTF, a method incorporates both features has been proposed, update by layers. This method focuses on one layer at a time, while keeping other layers unchanged, then 
uses all related light rays to update the selected layer [13]. The process is illustrated as below.

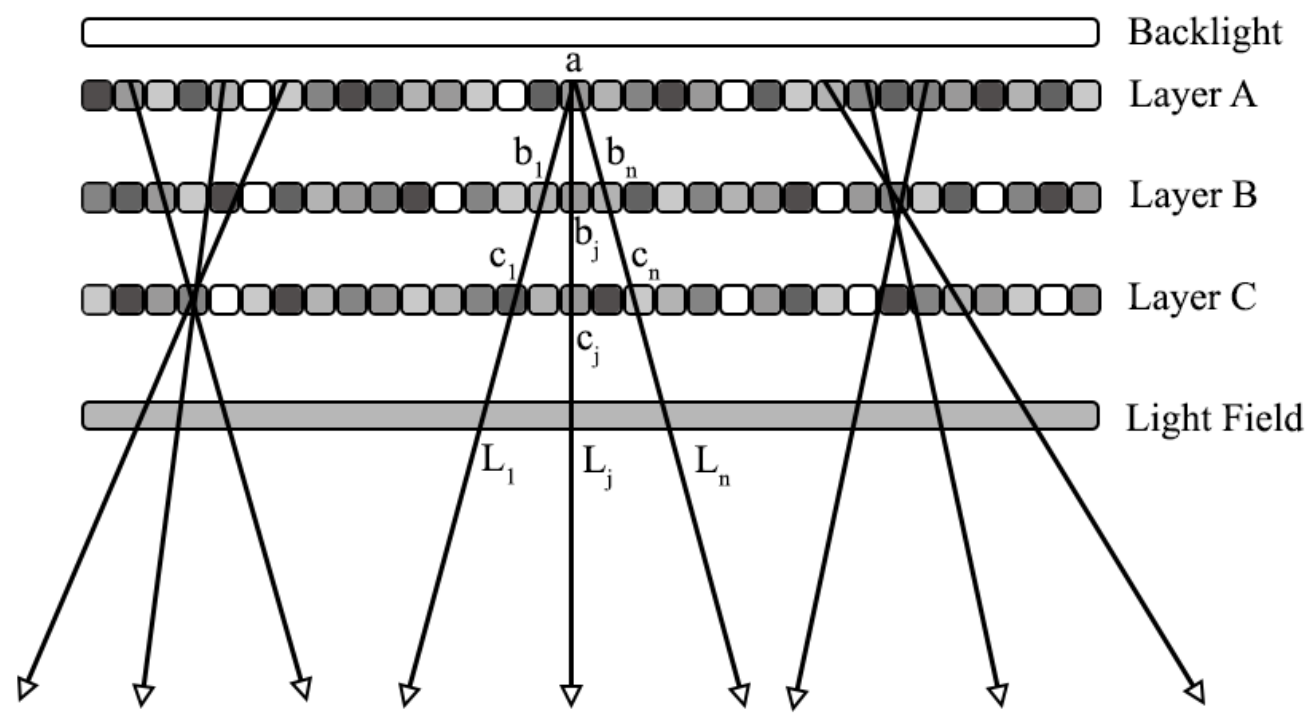

Figure 11 Illustration of update by layer method using $1 D$ slices of three-layer structure. (Center) Rear layer A is focused, and the intersection pixel values are marked in lower cases, where $n$ is the total number of views (only 3 are drawn in image), and $j$ is any number between 1 and $n$. (Left) The case of focusing on layer C. (Right) The case of focusing on layer B. L denotes the target light field value of that perspective. All pixel values range from 0 to 1 .

Take focusing on layer A for example. If we trace one ray that passes through intersections marked as $a, b_{j}, c_{j}, L_{j}$ in the image, the difference between emitted light field and the target light field can be written as:

$$
\text { error }=L_{j}-a \times b_{j} \times c_{j}
$$

If we consider all rays like this from layer $\mathrm{A}$, the minimum total error of the current reconstructed light field is:

$$
\min _{a} E(a)=\sum_{j=1}^{n}\left(L_{j}-a \times b_{j} \times c_{j}\right)^{2}
$$

where pixel value $a$ is limited between 0 and 1 to make calculation consistent. 
To make E(a) minimal, we can use its derivative and force it to be zero.

$$
\frac{d E(a)}{d a}=\sum_{j=1}^{n} 2\left(L_{j}-a \times b_{j} \times c_{j}\right) \times\left(b_{j} \times c_{j}\right)=0
$$

Then we can obtain the rule to update layer A.

$$
a^{\prime}=\frac{\sum_{1}^{n} L_{j} \times b_{j} \times c_{j}}{\sum_{1}^{n}\left(b_{j} \times c_{j}\right)^{2}}=\frac{\sum_{1}^{n} L_{j} \times b_{j} \times c_{j}}{\sum_{1}^{n}\left(\widehat{L_{j}} / a\right) \times\left(b_{j} \times c_{j}\right)}=a \times \frac{\sum_{1}^{n} L_{j} \times b_{j} \times c_{j}}{\sum_{1}^{n}\left(\widehat{L_{j}} \times b_{j} \times c_{j}\right)}
$$

Where $L_{j}$ is the target light field ray value, and $\widehat{L_{J}}$ is the reconstructed light field ray value $\left(\widehat{L_{j}}=a \times b_{j} \times c_{j}\right)$, and $a^{\prime}$ is the new value of $a$. Value of $a$ and $a^{\prime}$ is always between 0 and 1 in update iterations, and same rule applies to $b$ and $c$ as well when updating layer B and C.

In this way, we can write the update rule of all three layers:

$$
\left\{\begin{array}{l}
a^{\prime} \rightarrow a \times \frac{\sum_{1}^{n} L_{j} \times b_{j} \times c_{j}}{\sum_{1}^{n}\left(\widehat{L_{j}} \times b_{j} \times c_{j}\right)}, 0 \leq a^{\prime} \leq 1 \\
b^{\prime} \rightarrow b \times \frac{\sum_{1}^{n} L_{j} \times a_{j} \times c_{j}}{\sum_{1}^{n}\left(\widehat{L_{J}} \times a_{j} \times c_{j}\right)}, 0 \leq b^{\prime} \leq 1 \\
c^{\prime} \rightarrow c \times \frac{\sum_{1}^{n} L_{j} \times a_{j} \times b_{j}}{\sum_{1}^{n}\left(\widehat{L_{J}} \times a_{j} \times b_{j}\right)}, 0 \leq c^{\prime} \leq 1
\end{array}\right.
$$

The equations above demonstrate a clear and intuitive method using simple calculations based on visual understanding of ray tracing to update the image layers for reconstructing light field [13]. The method was first implemented and run on CPU, which was probably slow and no detail is given. Cao later suggests using CUDA for acceleration, then achieved average time of 671 milliseconds for 3 iterations according to their result [18]. As this method mainly deals with pixel level calculation, it is suitable for parallel computing, like what Cao did using CUDA, but 
more optimization can be implemented to achieve higher speed. This thesis focuses on improvement of this method. 


\section{HPO Design}

\subsection{HPO Concept explanation}

As discussed previously, although the existing light field display prototypes have provided many solutions of reconstructing target light field using multiple layers, there are various drawbacks and limitations. The key problem in designing a light field display is to speed up the calculation of layer images, while improve or at least maintain the output visual quality.

In this proposed HPO light field display, only horizontal views are kept for acceleration of computation, while vertical views are abandoned. As viewers can always see two different horizontal perspectives in two eyes, 3D effect is still achieved.

One important feature of the previous light field displays is that they can diminish the vergence-accommodation conflict. Namely, users can focus freely without causing any fatigue, since there exists natural depth cue formed by the displays. This HPO design only reduce the number of vertical views, therefore, as long as there are enough number of horizontal views for both eyes, the feature is inherited by our display.

\subsubsection{Algorithm complexity analysis}

Current solutions take input of light field image set that contains both vertical and horizontal perspectives and try to reconstruct light field emitting views in all these angles. Therefore, the input of these algorithms must be full images to correlate 
pixels with all views. For example, when we use NMF to compute two layers from a $512 \times 384$ light field with 7 horizontal views without splitting, the $\mathrm{V}$ matrix is (512 $\times 384) *(512 \times 384)$ in dimension, and the $\mathrm{W}$ and $\mathrm{H}$ matrices are $(512 \times 384) \times 1$ and $1 \times(512 \times 384)$ respectively. For SART, in each update of one layer, we have to do ray casting of $512 \times 384$ rays for each pixel. The amount of calculation is the main reason these algorithms consumes long time for computation.

Our method, HPO algorithm, decouple the horizontal pixels. If light rays of views only spread in one direction, there is no more correlation between lines of pixels. Therefore, the pixel lines can be split and processed individually, also in parallel. Since our stereoscopic vision is usually composed by two perspectives, left and right, thus the vertical views can be disposed to decouple the correlations, and only horizontal parallax is left. This can bring huge improvement in speed. If we split the full images into horizontal pixel lines, the calculation becomes decomposing $512 \times 512$ into $512 \times 1$ and $1 \times 512$. The $\mathrm{m} \times \mathrm{n}$ calculation becomes $\mathrm{m}$ only, and the theoretical acceleration can be up to $\mathrm{n}$ times.

For update by layer method, if images are split into pixel lines, the lines can be stored in a faster memory inside GPU, rather than the global memory, which will be introduced later. With much faster memory manipulation, computation can be greatly accelerated and real-time rendering can probably be achieved. Consequently, we propose a Horizontal Parallax Only (HPO) light field display to achieve this, and we focus on the update by layer method, as it is suitable for parallel computing and have greater potential for acceleration. 
One important issue must be stressed that HPO does not simply drop vertical views for slightly faster speed, such as from $7 \times 7=49$ views to 7 views, many more works must be modified in algorithm to achieve considerable acceleration in computation. The details are given in next chapter.

To make an HPO light field display, special design of optical and mechanical components is needed.

\subsection{Optical design}

The HPO light field display system have several optical components: collimated backlight, horizontal diffuser, stacked LCD panels, and vertical diffuser. A two-layer configuration system is shown below.
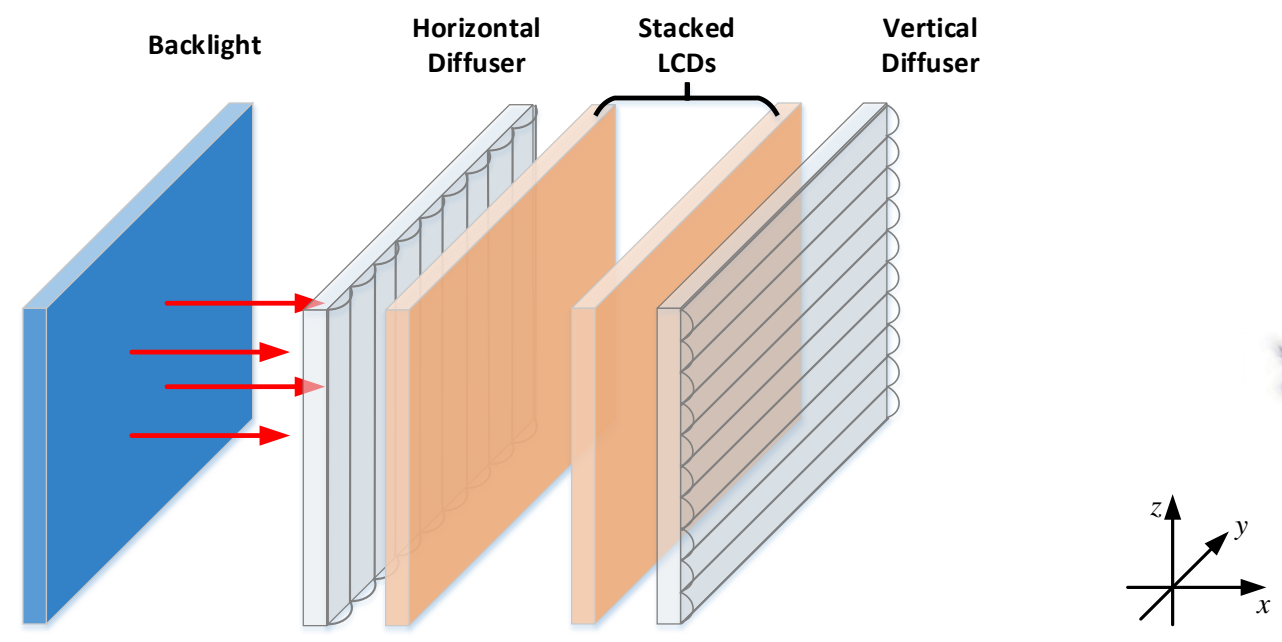

Figure 12 Two-layer HPO system configuration. From left to right: Collimated backlight, horizontal diffuser, stacked LCDs, vertical diffuser.

The collimated backlight emits light rays in the direction perpendicular to the horizontal diffuser plane, and it is designed as below. 

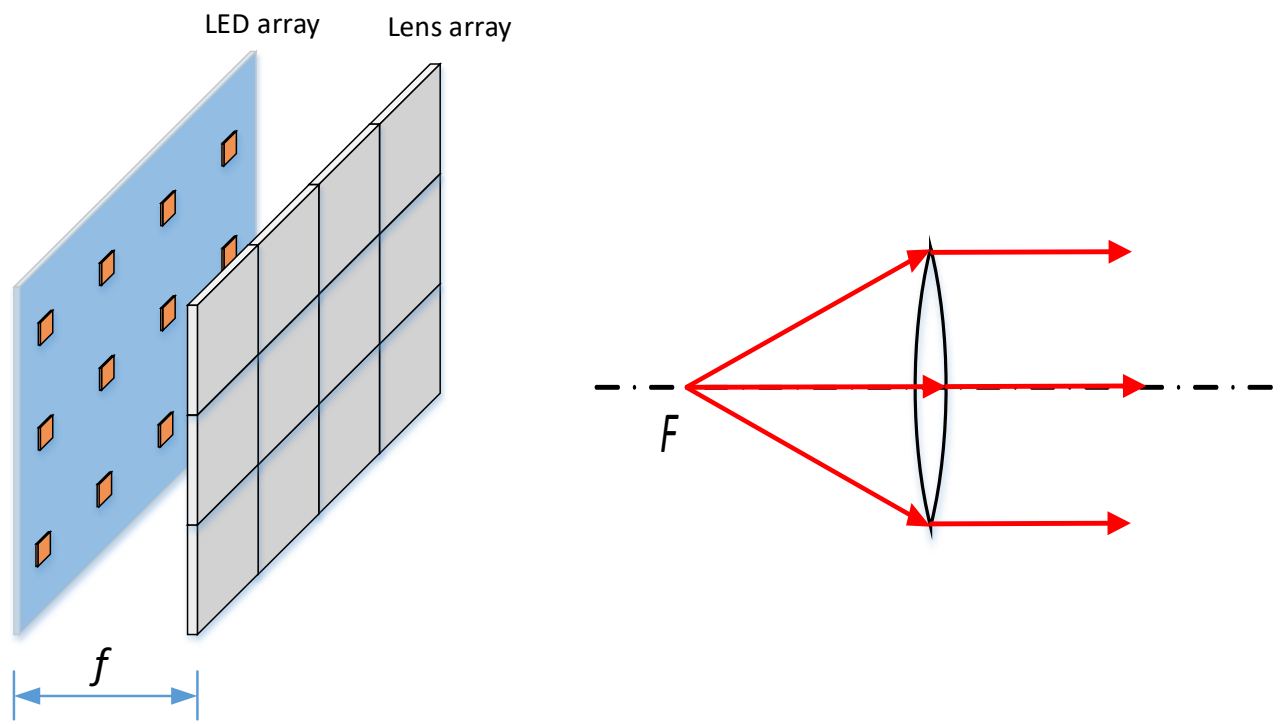

Figure 13 Configuration of collimated backlight. (Left) Light emitted from LED array will enter the lens array. (Right) The lens array can convert uncollimated light rays into parallel light rays.

The light rays go into the horizontal diffuser at rear of the LCD panels will diverge and cover a certain angle, thus shed light on a few pixels. In this way, each pixel on the rear LCD is only correlated with a number of pixels on the front panel of the same height, as shown in image below.
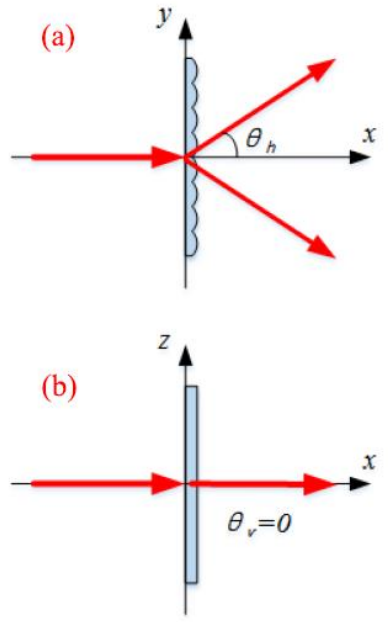

(c)

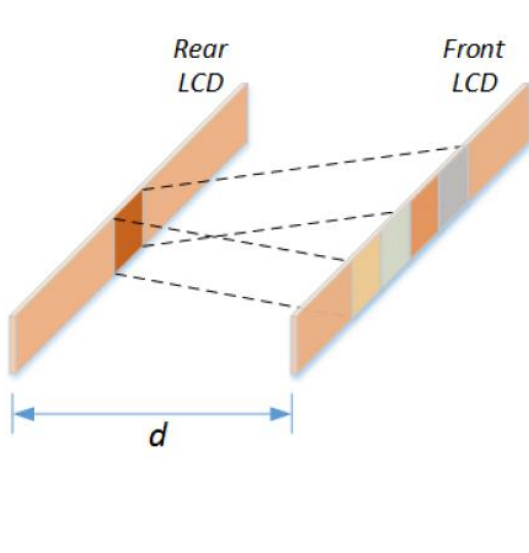

(d)
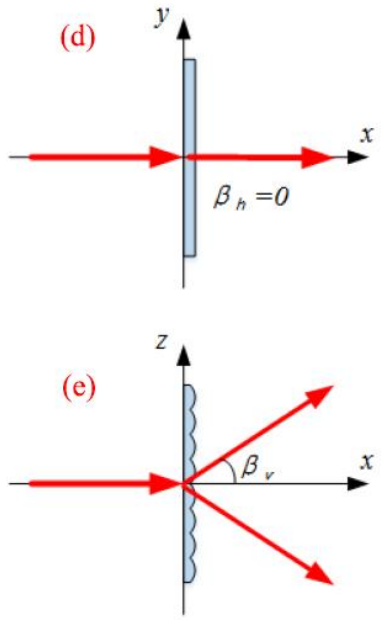

Figure 14 Illustration of light ray in HPO system. (a) Ray distribution at horizontal diffuser from top view. (b) Ray distribution at horizontal diffuser from side view. (c) Ray from one pixel on rear panel diverges into a range of rays and cover several pixels on front panel. $(d)$ 
Ray distribution at vertical diffuser from top view. (e) Ray distribution at vertical diffuser from side view. Note that the axis in this image follows the coordinate system shown in HPO system configuration.

\subsection{Mechanical design}

To achieve the optical design of the HPO system, mechanical components are drawn for prototyping.

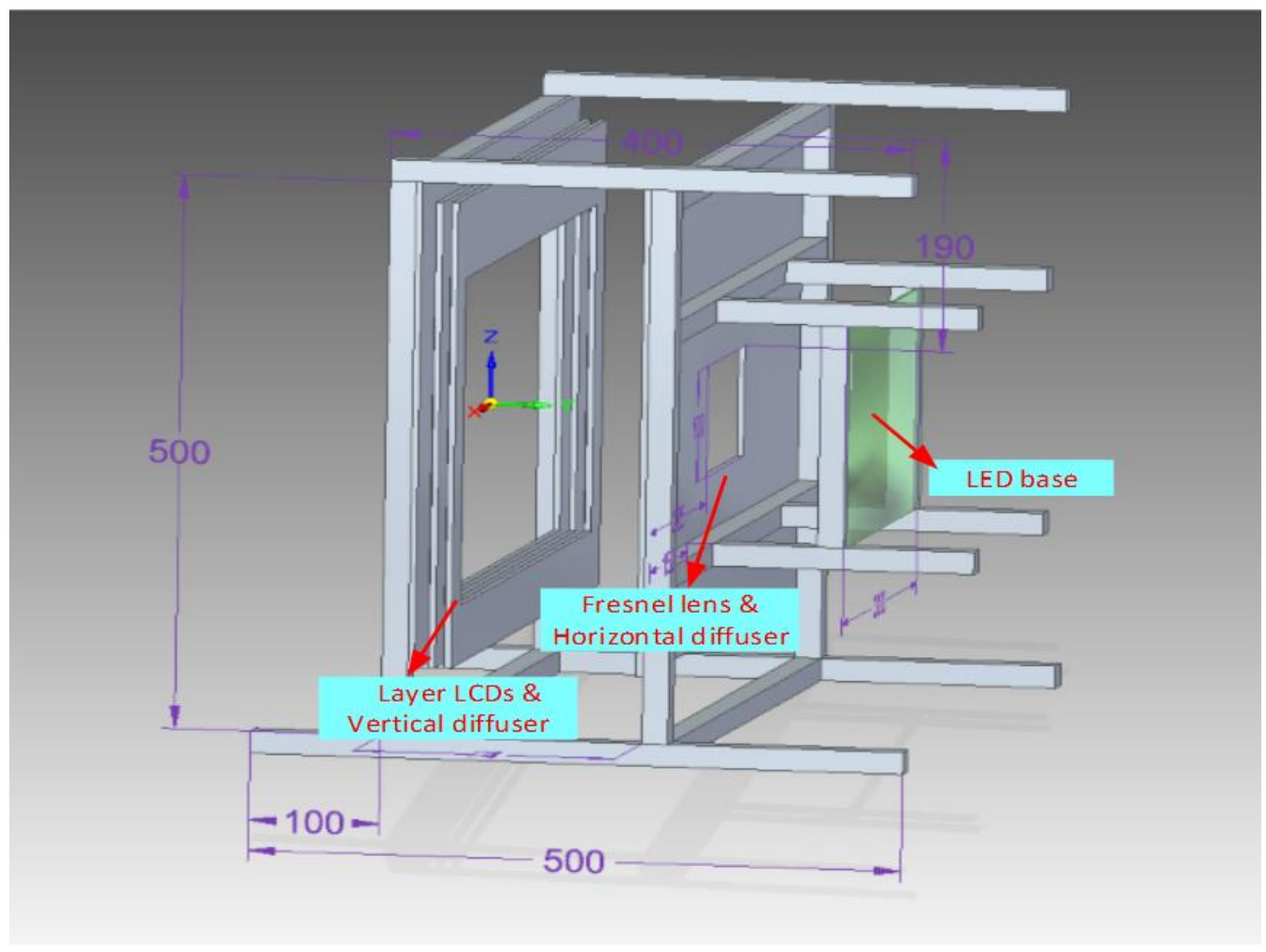

Figure 15 Mechanical design of HPO system. (From right to left) LED base as backlight, Fresnel lens to collimate light, Horizontal diffuser to diffuse light horizontally, Layer LCDs, Vertical diffuser to diffuse light vertically. The dimensions marked in image are all in millimeters.

The LCD panels to be used for this system are dissembled screens from Asus VG248QE monitors. 
As of the moment of writing this thesis, the designed frames are still in production. Therefore, no final product can be shown, and we shall discuss simulation results in results part. 


\section{HPO Algorithm development}

In all previous works, light field images are processed using full image, which makes parallel computing inefficient or difficult. HPO does not simply mean reducing vertical perspectives of input light field, additional creative optimizations are applied and dramatically accelerate the computation of layer images.

This part shows how an innovative algorithm to accelerate layer image generation using CUDA programming is developed and optimized.

\subsection{CUDA parallel computing}

In image processing, a very intuitive way to accelerate calculation is splitting the task into smaller pieces and use multiple threads to process these pieces, namely parallel computing. There exist several tools several tools can implement such operations, some utilizes CPU, some uses GPU, while some can employ both. As GPUs today have much greater number of micro cores (called kernels in CUDA) than CPU, thus more suitable for parallel computation. Among those tools mainly utilize GPU, CUDA from NVIDIA has been proved as one of the most efficient one [19]. The parallel operations are passed from CPU to GPU for execution and happen in kernels, which are defined in the key component of a CUDA program, kernel code. 


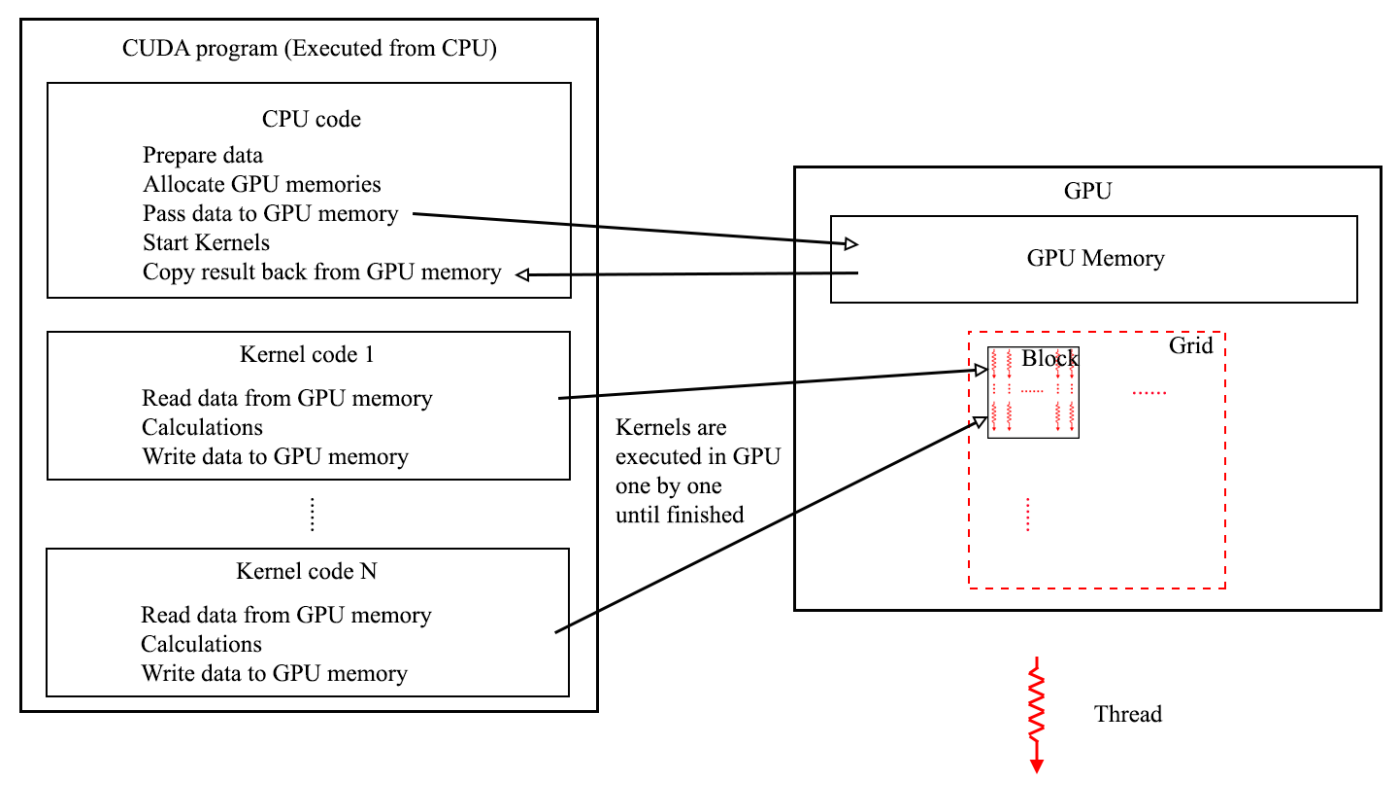

Figure 16 CUDA program structure. There can be multiple kernel codes. Kernel codes are passed to GPU for execution sequentially, i.e. kernel code 2 will only start when kernel code 1 finishes. Each kernel code is run in necessary number of kernels (defined at kernel call in CPU code).

To process an image, for example, a popular method using CUDA is do calculation at pixel level, using one kernel for each pixel, as known as threads. An illustration of blurring an image is drawn below. 


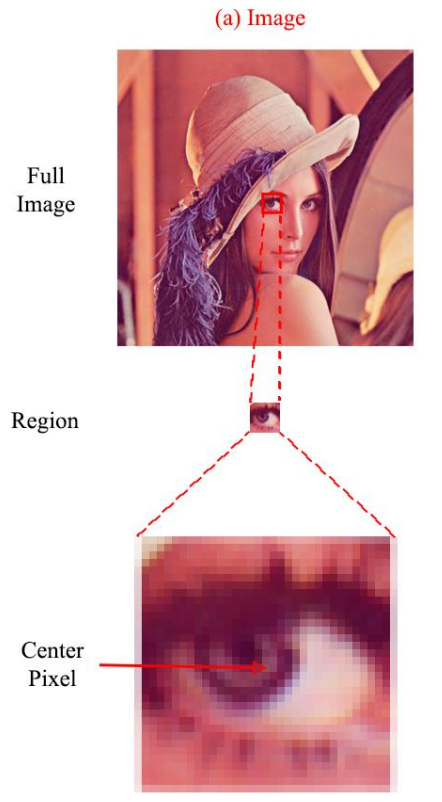

(b) CUDA
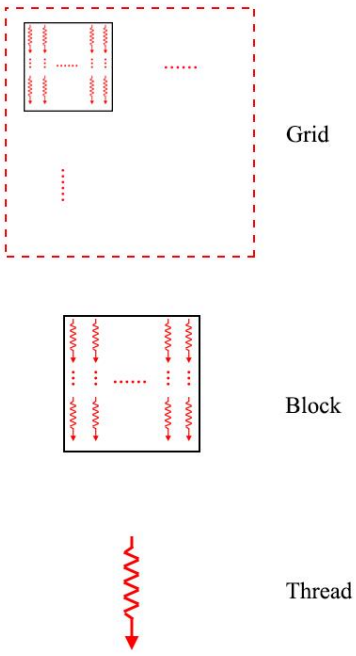

Figure 17 Example of blurring an image with CUDA. (a) Image level illustration: Full image is divided into many regions, and these regions are further split into pixels, where each pixel is blurred based on surrounding pixels. (b) Implementation in CUDA: A grid structure is responsible for whole image, and it contains many small blocks that deal with small regions. The basic unit in CUDA is thread, and each of it calculate the average of a number of surrounding pixel values and reflect the result on center pixel. Each thread is executed in a separate kernel. The image is a photo called "Lena" from the USC-SIPI image database [20].

As we can see from the visual explanation, CUDA is a powerful tool for image processing. However, the performance, especially the computation speed, heavily depends on optimization, such as how to organize blocks and threads and memory management, which is the key innovation point of the algorithm of this HPO system.

\subsection{Development}

\subsubsection{Early development}

Following the previous part's idea, it is easy to figure out how to implement the update by layer method for computing image layers from input light field image set using CUDA. A two-layer configuration is used for clearer understanding. 


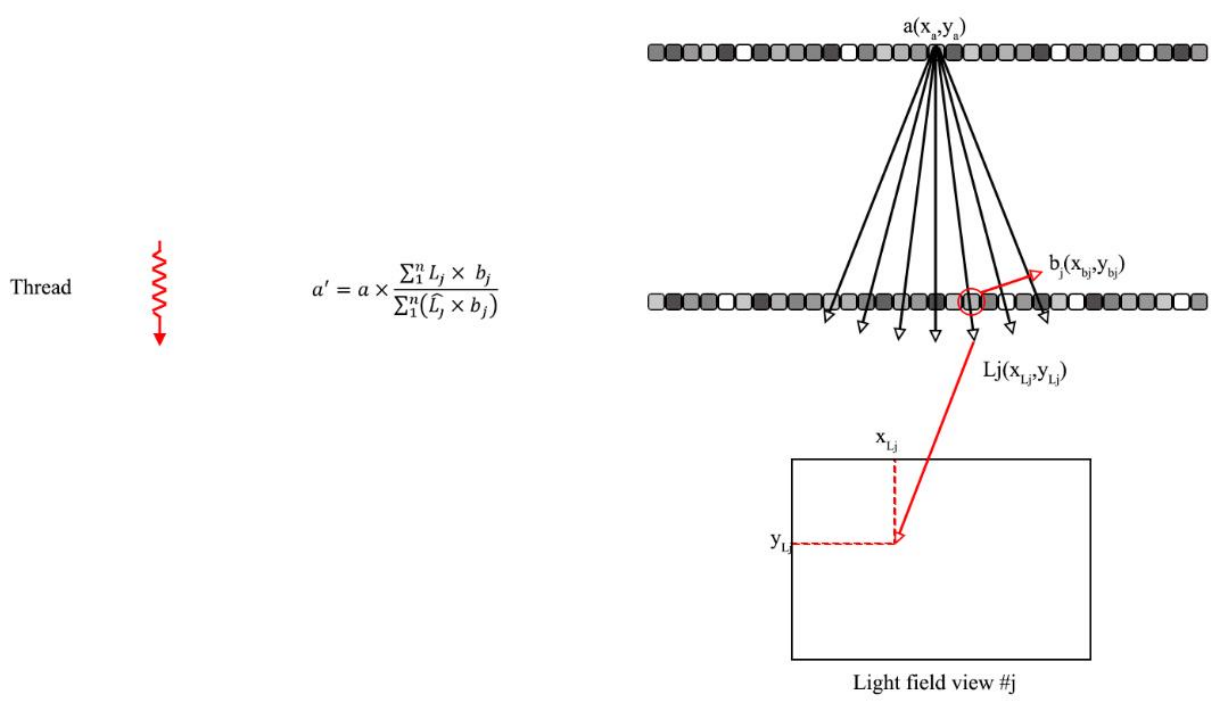

Figure 18 Update by layers in CUDA. Every thread is responsible for updating a pixel value $a$ on one layer with coordinates $\left(x_{a}, y_{a}\right)$. The thread calculates rays' directions to find intersections. One ray of view \#j has intersections with another layer and target light field, labeled $b_{j}$ and $L_{j}$, with coordinates $\left(x_{b j}, y_{b j}\right)$ and $\left(x_{L j}, y_{L j}\right)$ respectively. The pixel values are used according to the equation and pixel $a$ is updated.

This is a straight forward implementation. We implemented this algorithm using CUDA, and the pseudocode of kernel code is given as below. 


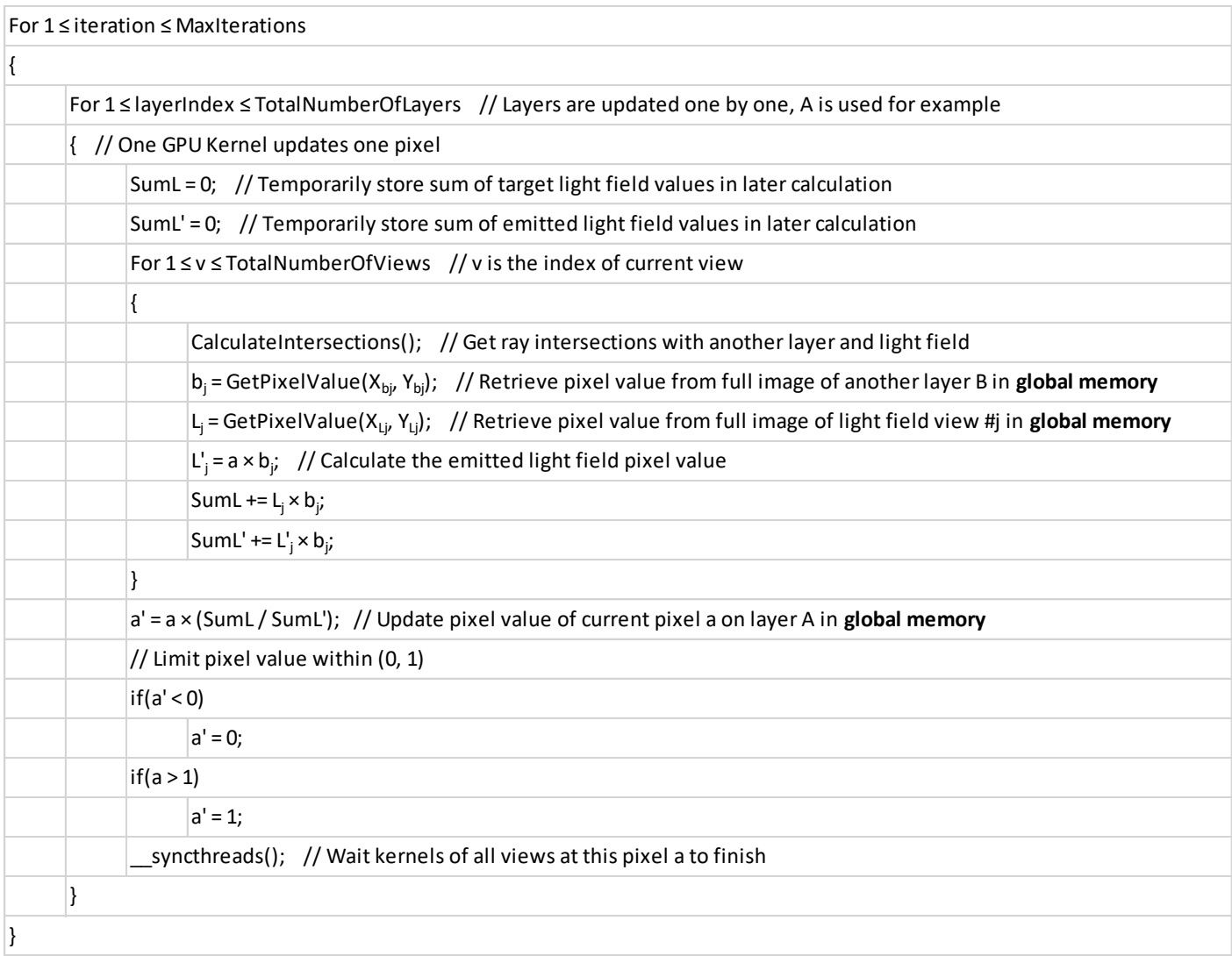

Figure 19 Pseudocode in CUDA, early version. Access to global memory is marked to be explained later.

As mentioned in the pseudocode, the intersections of rays are calculated repeatedly within each kernel update. In the early development stage, all intersections were calculated beforehand in CPU and copied to GPU memory, and kernel fetched the intersections from GPU memory each time. The overall calculation time of one iteration was too long, more than 500 milliseconds, which was obviously unsatisfying. When the calculation of intersections was moved to kernel code, the execution time becomes lower by half. This was the moment we noticed the impact of GPU memory access on execution time.

With the early version of algorithm described above, the intuitive method was verified to be working, but on the basis of full image and perspectives of all both 
horizontal and vertical direction. When we abandoned the vertical views, the reduction in processing time was not impressive, just as what we expected. More modifications must be made to boost the performance.

\subsubsection{Optimizations}

Since vertical views were disposed, there is no more correlation between horizontal pixel lines. As a result, we had much more freedom in playing with smaller fractions of images. The first thing we did is on memory management.

To achieve faster memory manipulation, the structure of GPU memory must be studied first.

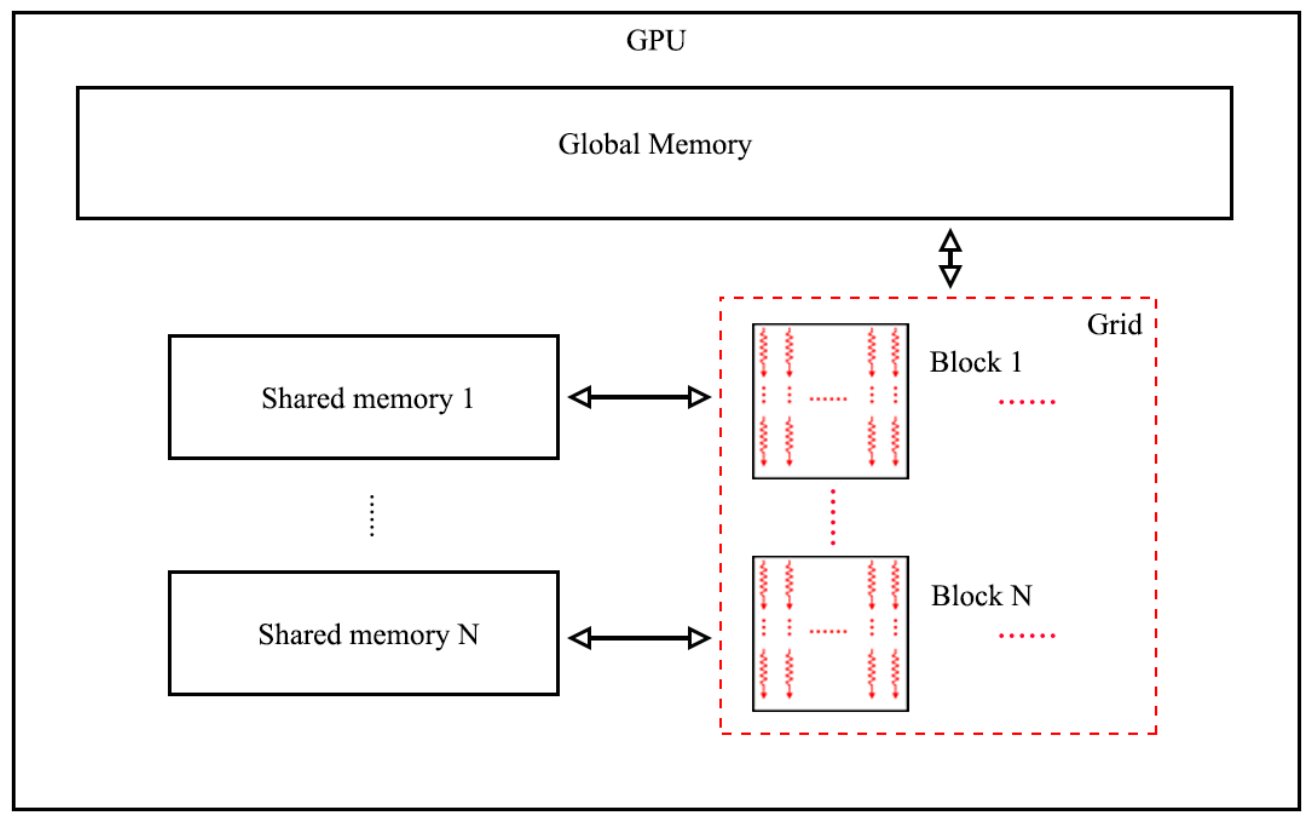

Figure 20 GPU memory structure in CUDA. There are three types of memories can be utilized by CUDA. Global Memory: A very large memory that all threads in all blocks can access, data inside is kept after kernel executions. Shared Memory: A smaller memory shared by threads within the same block, data inside is deleted after one kernel code finishes. Local Memory: The smallest memory used by each thread, one thread cannot access another one's local memory. 
As shown above, a CUDA can manipulate three kind of memories. While global memory is commonly used in other tools like OpenGL to store data like textures, shared memory and local memory are not usually accessible by these tools, since they automatically handle the memory according to actual use. However, automatic management is not always optimal, especially when these tools are not used for their original purposes (3D scene rendering for the case of OpenGL). Therefore, with better memory management, the light field computation can be accelerated using CUDA.

The three mentioned types of GPU memories have different read and write speed for threads. The local memory is fastest, but the size is small, since threads are most used for simple calculations. The shared memory is slightly slower, but still much faster than the global memory, with larger size than local memory. While accessing shared memories takes 20 to 40 clock cycles (one integer or floating point calculation takes about 6 clock cycles), reading or writing to the global memory costs 400 600 clock cycles, as we tested and measured. Therefore, the early version was slow.

To make access faster, it is necessary to move intermediate results within loops from global memory to shared memory. However, the size of shared memory is limited, 49152 bytes. The size of one single $512 \times 384$ image in RGB color channels with pixel values stores as floating number between 0 and 1 consumes $512 \times 384 \times 3 \times$ $4=2359296$ bytes, which is too large for shared memory, let alone the fact that there is light field image set and multiply layers. we cannot simply store the whole images into shared memory for faster access. 
Since horizontal pixel lines are decoupled, full images can be split into smaller slices and stored in shared memory. A $512 \times 384$ image is divided into 384 horizontal pixel lines with 512 pixel values, and this operation is done for both light field image set and layers. These lines are copied to shared memory of blocks. In each block of threads, threads read and write pixel values of light field images and layer images on the same horizontal line.

In the early development, according to figure 18, the equation 17 can be rewritten as:

$$
\left\{\begin{array}{l}
a^{\prime} \rightarrow a \times \frac{\sum_{1}^{n} L_{j} \times b_{j}\left(x_{b_{j}}, y_{b_{j}}\right)}{\sum_{1}^{n}\left(\widehat{L_{J}} \times b_{j}\left(x_{b_{j}}, y_{b_{j}}\right)\right)}, 0 \leq a^{\prime} \leq 1 \\
b^{\prime} \rightarrow b \times \frac{\sum_{1}^{n} L_{j} \times a_{j}\left(x_{a_{j}}, y_{a_{j}}\right)}{\sum_{1}^{n}\left(\widehat{L_{J}} \times a_{j}\left(x_{a_{j}}, y_{a_{j}}\right)\right)}, 0 \leq b^{\prime} \leq 1
\end{array}\right.
$$

Now with no more vertical views exist and use of shared memory, the equation becomes

$$
\left\{\begin{array}{l}
a^{\prime} \rightarrow a \times \frac{\sum_{1}^{n} L_{j} \times b_{j}\left(x_{b_{j}}\right)}{\sum_{1}^{n}\left(\widehat{L_{J}} \times b_{j}\left(x_{b_{j}}\right)\right)}, 0 \leq a^{\prime} \leq 1, \text { within same block } \\
b^{\prime} \rightarrow b \times \frac{\sum_{1}^{n} L_{j} \times a_{j}\left(x_{a_{j}}\right)}{\sum_{1}^{n}\left(\widehat{L_{J}} \times a_{j}\left(x_{a_{j}}\right)\right)}, 0 \leq b^{\prime} \leq 1, \text { within same block }
\end{array}\right.
$$

This does not only mean that we no longer have to calculate y axis, but more importantly means we can access shared memory quickly of the block to update pixel values, instead of lengthy query at GPU global memory.

In the finalized algorithm, we adopted the discussed optimization, as shown in the pseudo code below. 


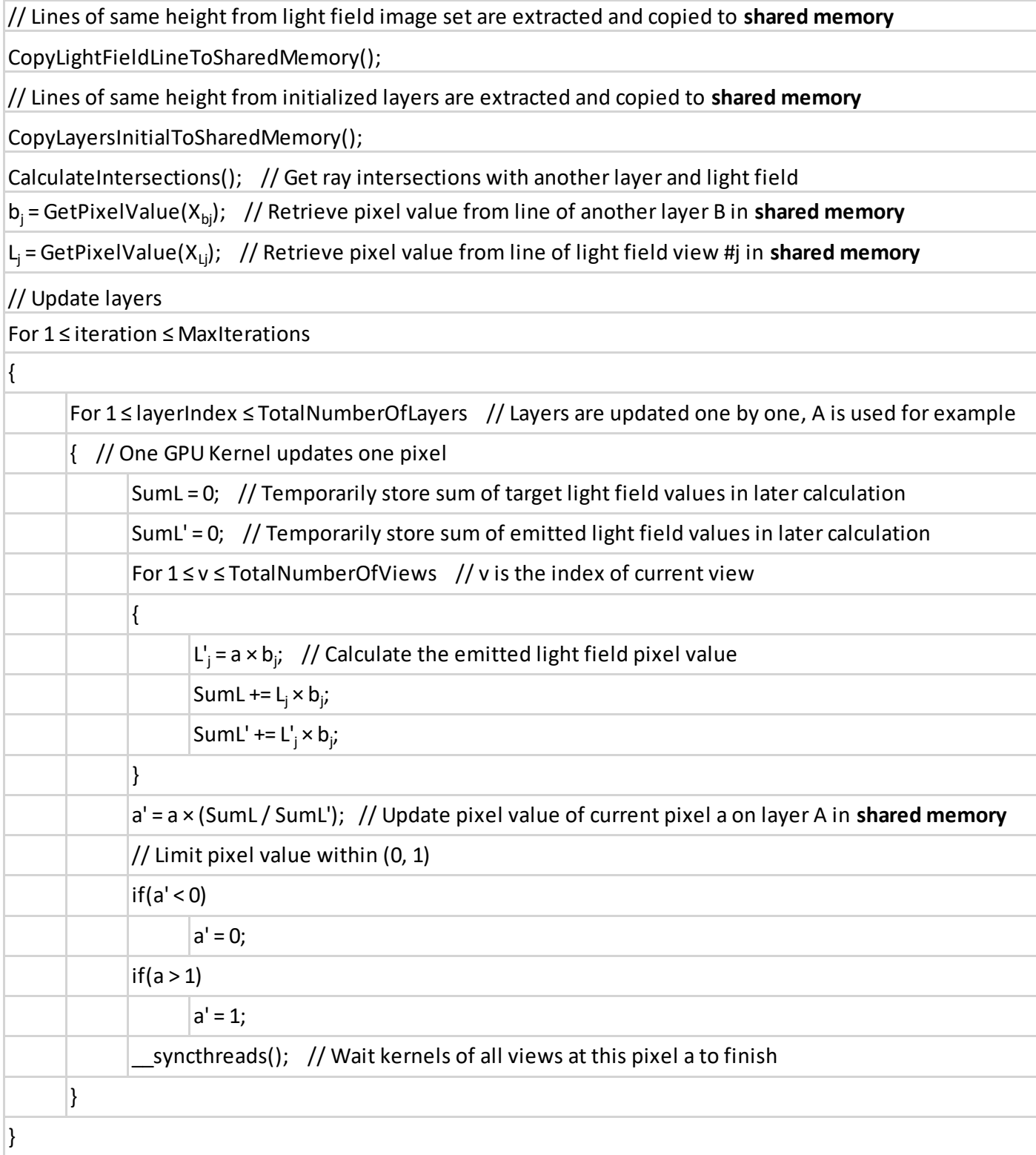

Figure 21 Pseudo code in CUDA, finalized version.

There are some optimizations not reflected in the pseudo code, but actually implemented in program.

In memory access, another factor that may influence performance is type of access. There are three types of access, coalesced, striped, and random. Coalesced access refers reading or writing neighbor elements in memory together and it is fastest, 
while striped access means fetching or assigning values to every other element in memory, i.e. access $1^{\text {st }}$ then $3^{\text {rd }}, 5^{\text {th }}$, and so on, and this is slower. The slowest is random access. We rearranged the light field image set that pixels of same position but belong to different views are grouped together, to make access coalesced.

Other than the memory optimization discussed above, a few other factors were also considered. For example, the full color images have three channels of red, green, and blue. These channels are executed in parallel for acceleration, too, by carefully locating them in different position within the grid and use proper block and thread indexing.

\subsection{Theoretical advantages}

The algorithm is based on the new HPO light field design. It can possibly improve the computation speed greatly. For generating image layers of a $512 \times 384$ light field with 7 horizontal views, the difference using previous methods and HPO can be as high as 384 times. Of course, considering the data accessing time, the actual performance improvement will probably not be this much, possibly less than 100 times faster.

While the computation speed potentially increases, the image quality should be similar, since the core updating rule is maintained.

In next chapter, these possibilities are examined by a series of assessments. 


\section{Results and Discussion}

To verify the mentioned advantages of HPO design and algorithm, various comparisons were conducted. As no detailed result of NMF method was been given regarding computation speed, which is the key improvement of HPO, the evaluations were mainly made with HPO and SART method, as the we managed to reproduce the SART program. For NMF, an indirect comparison in speed was made.

To be fair for the two methods, they were executed on the same computer. The specification of the computer is given below.

\begin{tabular}{|c|c|c|}
\hline Hardware Name & Model & Details \\
\hline Operating System & Windows 10 Pro & Build 15063 \\
\hline CPU & i7-6700K & CPU Clock 4.0GHz \\
\hline Memory (RAM) & Kinston DDR4 & 16GB, 2133MHz \\
\hline GPU & GTX 1080 & $\begin{array}{c}\text { GPU Clock 1785MHz, Memory Clock 1251MHz } \\
\text { GPU Memory 8GB, NVIDIA driver version 378.92 }\end{array}$ \\
\hline
\end{tabular}

Figure 22 PC configuration

Hardware that may affect the performance are all specified in the figure. Future works should consider these factors in comparison of results.

Two light field image sets, Dice and Messerschmitt, were obtained from the Synthetic Light Field Archive of MIT Media Lab data [7]. Both of them have 7 horizontal perspectives with $10^{\circ}$ FOV. PSNR and Structural Similarity (SSIM) are measured using MATLAB, and all given results are average values of multiple trials. 


\section{1. $\quad$ Reconstructed light field}

The original light field 2D image set and the resultant reconstructed light field images together with layer images are compared in this part. The setup of both SART and HPO programs in this comparison are given as follows:

\begin{tabular}{|c|c|}
\hline Item & Data \\
\hline Perspectives & 7 \\
\hline Resolution & $512 \times 384$ \\
\hline Pixel Pitch & $0.02767 \mathrm{~mm}$ \\
\hline Layer Gap & $16 \mathrm{~mm}$ \\
\hline Light Field Position & On Front Layer \\
\hline Number of Iterations & 10 \\
\hline
\end{tabular}

Figure 23 Parameters in both SART and HPO program

The comparison regarding Dice light field is as shown below:
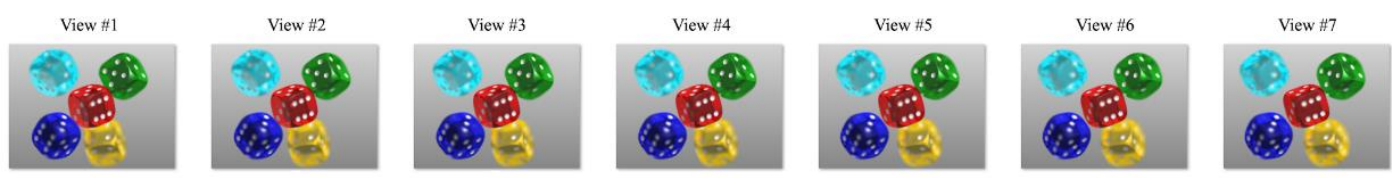

Original Light Field
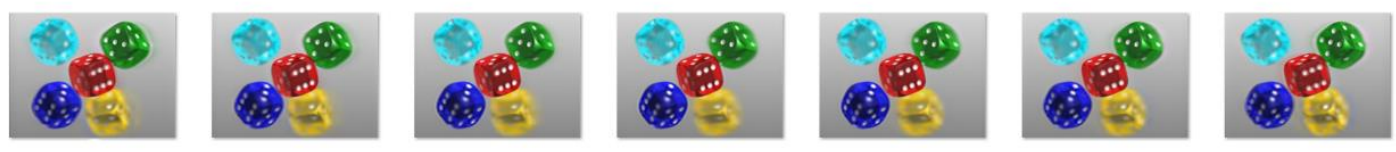

Reconstructed Light Field of SART

Time $1153 \mathrm{~ms} \quad$ PSNR $28.41 \mathrm{~dB} \quad$ SSIM 0.9367
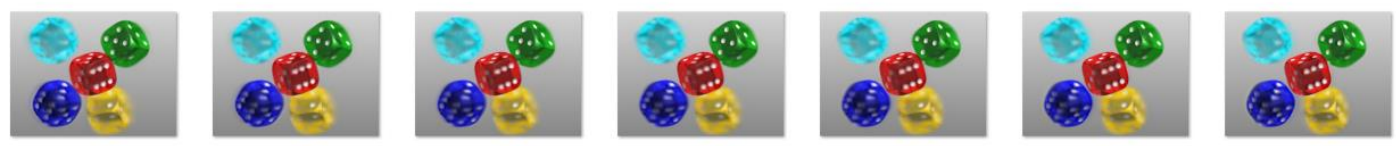

Reconstructed Light Field of HPO

Time $27 \mathrm{~ms} \quad$ PSNR $27.67 \mathrm{~dB} \quad$ SSIM 0.9567
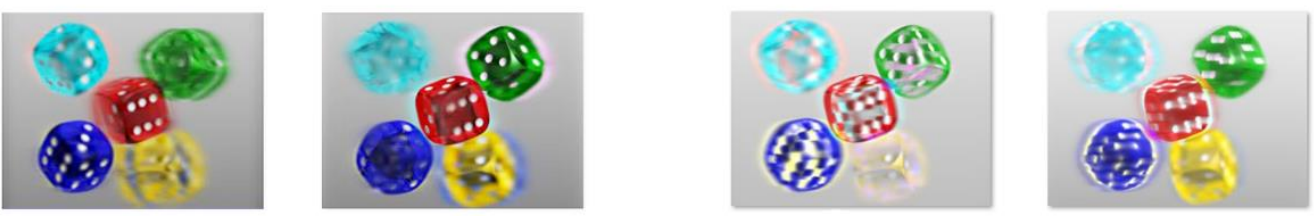

Layers of SART

Layers of HPO 
Figure 24 Original light field and reconstructed light fields of Dice with time, PSNR and SSIM

The comparison regarding Messerschmitt light field is as shown below:
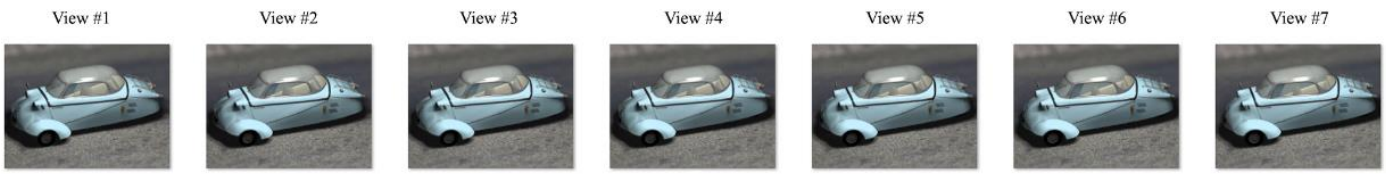

Original Light Field
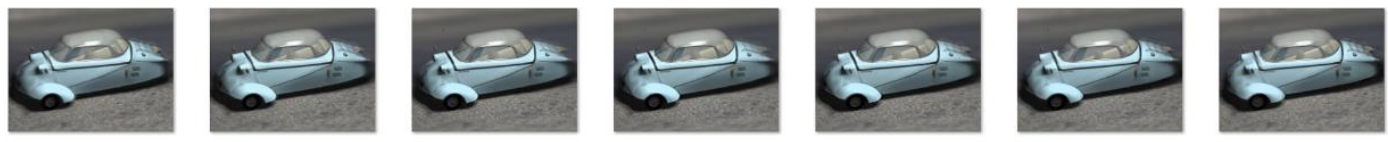

Reconstructed Light Field of SART

Time $1180 \mathrm{~ms} \quad$ PSNR $31.27 \mathrm{~dB} \quad$ SSIM 0.9265
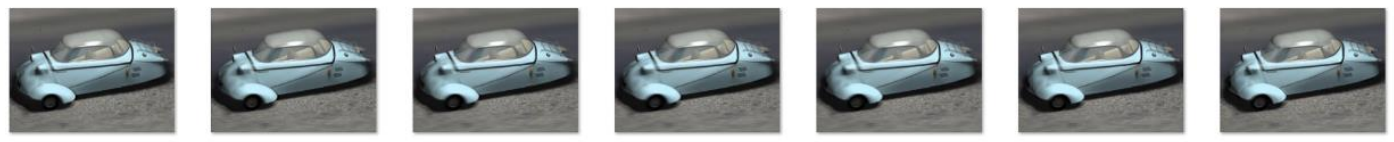

Reconstructed Light Field of HPO

Time $27 \mathrm{~ms} \quad$ PSNR $29.16 \mathrm{~dB} \quad$ SSIM 0.9130
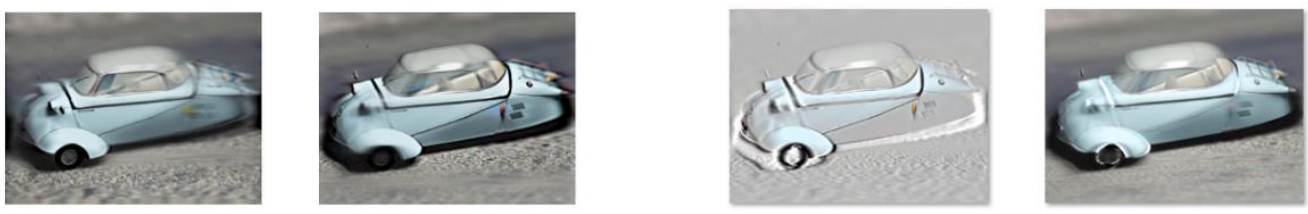

Layers of SART

Layers of HPO

Figure 25 Original light field and reconstructed light fields of Messerschmitt with time and PSNR.

Two more datasets with same setup were examined, and the result is shown in the table below.

Table 1 Extra data sets tested.

\begin{tabular}{|c|c|c|c|c|}
\hline Dataset Name & Method & Time & PSNR & SSIM \\
\hline \multirow{2}{*}{ Red Dragon } & SART & $1162 \mathrm{~ms}$ & $29.36 \mathrm{~dB}$ & 0.9312 \\
\cline { 2 - 5 } & HPO & $27 \mathrm{~ms}$ & $27.87 \mathrm{~dB}$ & 0.9227 \\
\hline \multirow{2}{*}{ Happy Buddha } & SART & $1176 \mathrm{~ms}$ & $30.24 \mathrm{~dB}$ & 0.9236 \\
\cline { 2 - 5 } & HPO & $27 \mathrm{~ms}$ & $28.13 \mathrm{~dB}$ & 0.9144 \\
\hline
\end{tabular}


The reconstructed light fields by both methods have little visual difference, while layer images vary due to difference in algorithm. In all scenes tested, PSNR of SART is slightly higher than that of HPO, except SSIM of HPO in Dice is higher than that of SART. More detailed examination is needed to explain this. Since the two extra datasets shows similar results, they will not be used for next recursive and lengthy experiments for simplicity.

\subsection{PSNR across pixel lines}

As horizontal pixel lines are decoupled and no longer correlated, it is worth examining whether PSNR of each of these lines are consistent with the overall PSNR. This may give some hint on how HPO affects image quality, by looking for rows with inconsistent PSNR values. 


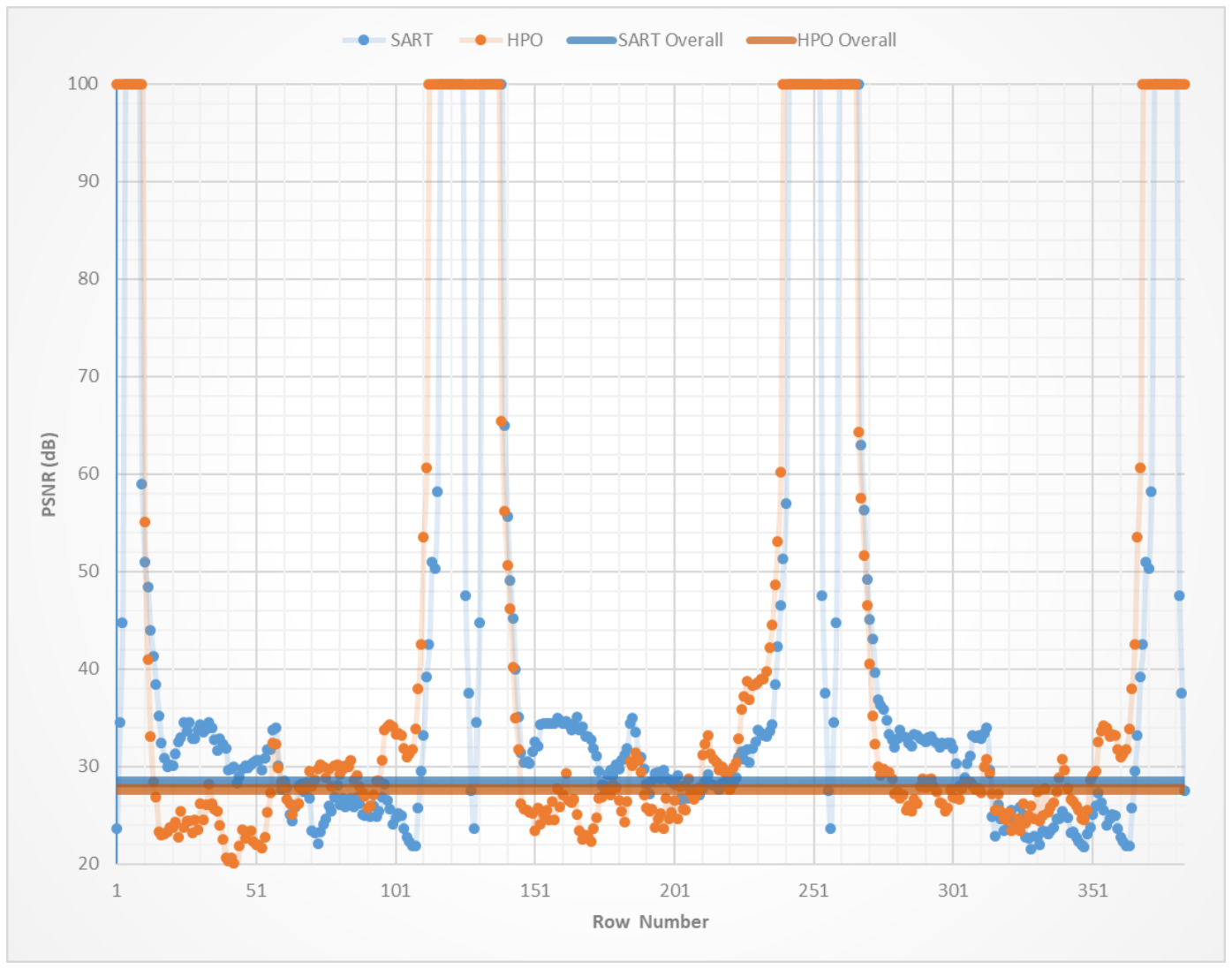

Figure 26 PSNR across lines in Dice reconstructed light fields

PSNRs of $100 \mathrm{~dB}$ in image actually have values of infinity, which means the sample is identical to the reference. 


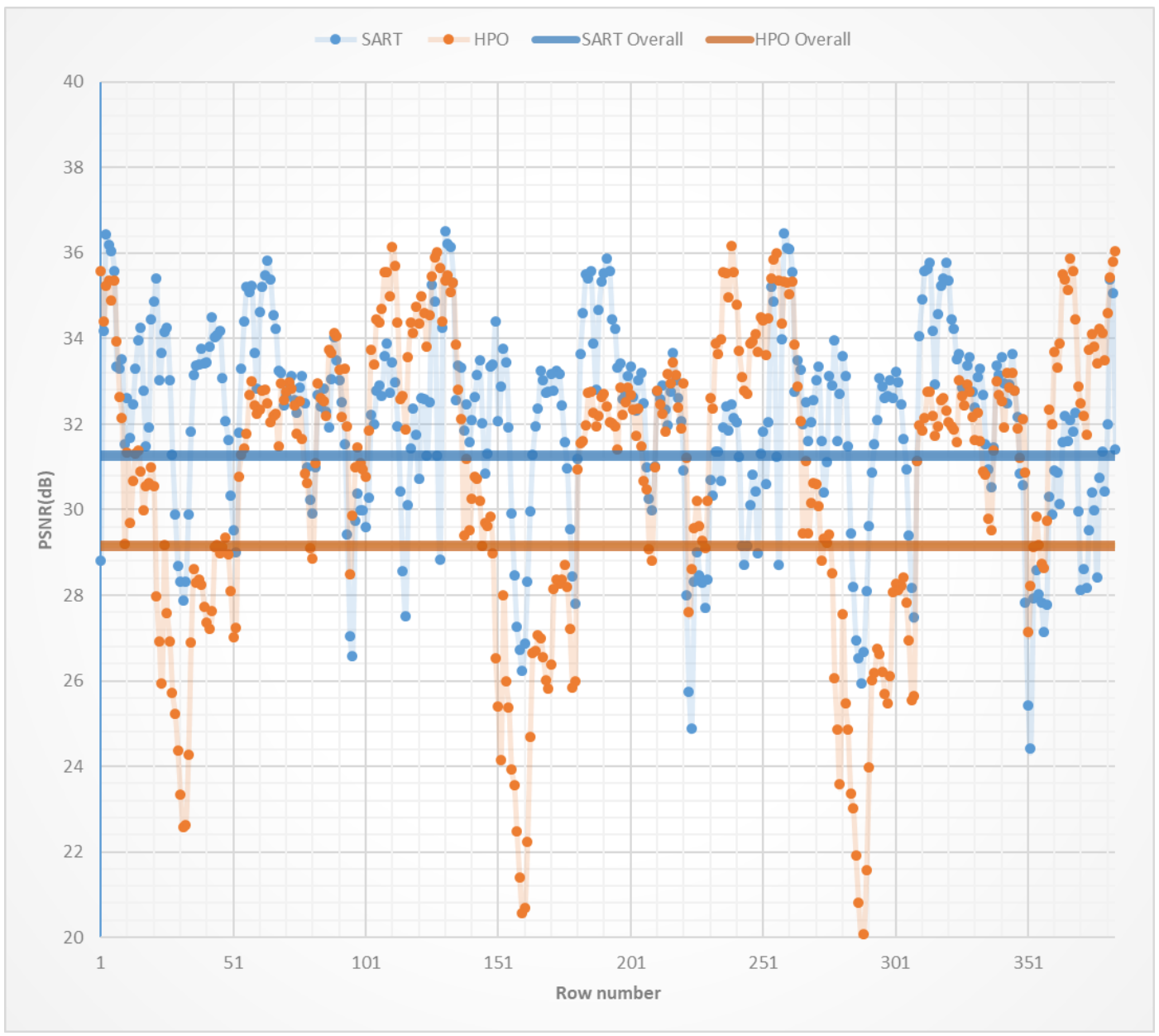

Figure 27 PSNR across lines in Messerschmitt reconstructed light fields

In Dice scene, both methods exhibit similar fluctuation across rows around overall PSNR value. It is noted that there are four peaks found in both methods indicating four ranges of pixel lines are perfectly reconstructed. By inspecting the content of such rows, two types of them can be found to explain the reason. First one is the row that shows static background, like the top ones and the bottom ones, while the second one is the row that only have insignificant change across perspectives, such as the rows of other two peaks.

On the other hand, HPO shows larger deviation from the overall value than SART method. The Messerschmitt scene has high-detailed car model and a more 
complicated background across views due to the unsmooth cement texture. We can probably deduce that HPO's performance slightly degrades in complex scenes. As for the fact that no peaks are observed in the Messerschmitt scene, it is possibly due to the changing background across views, since even far background demonstrates nonnegligible variation.

\subsection{Computation time vs number of iterations}

The main goal of this HPO method is to speed up computation, therefore the most important evaluation is undoubtedly the processing time used for updating layers. Computation times taken for different number of iterations from 1 to 100 for reconstruction of both light fields are shown for comparison. Times are measured against number of iterations.

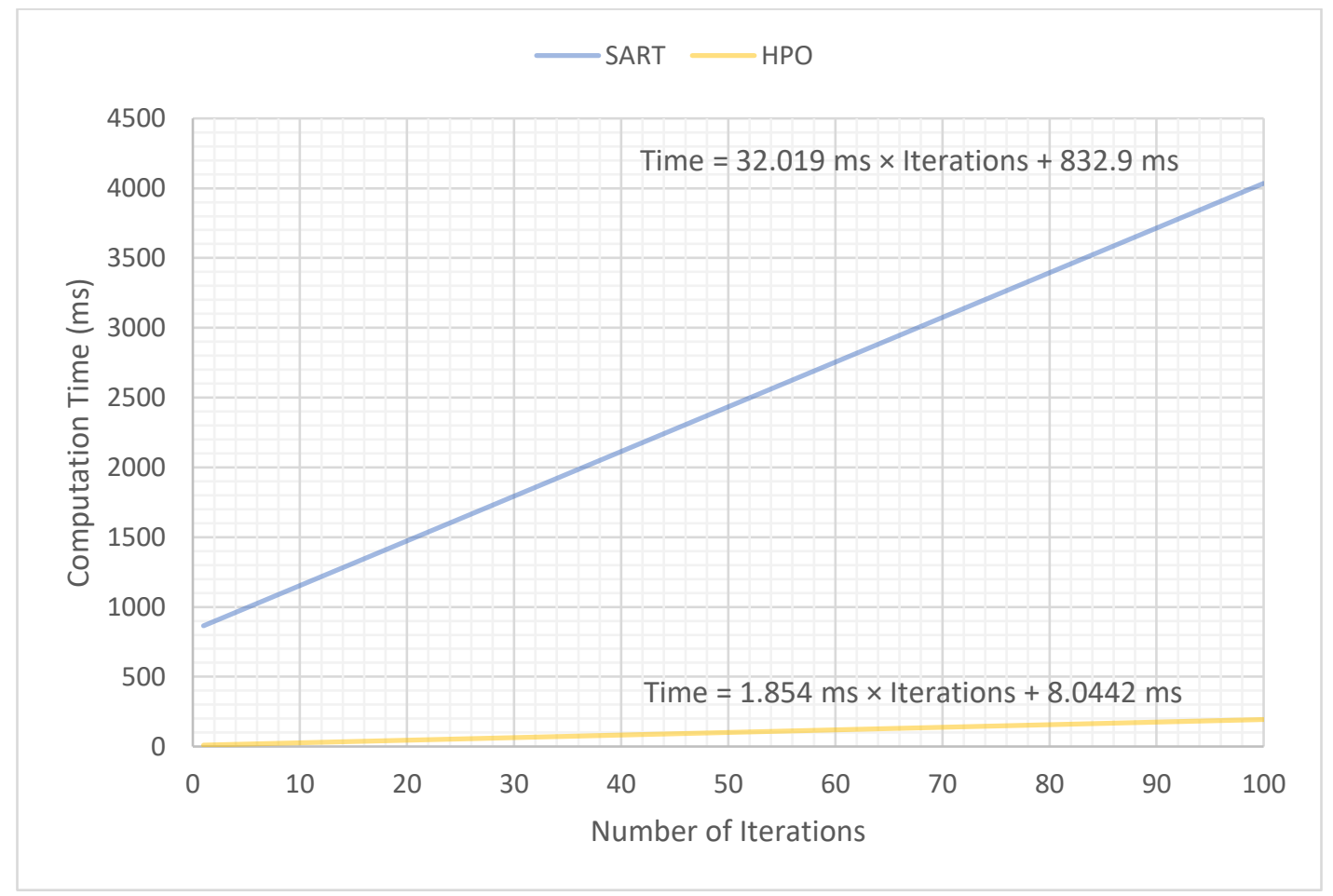

Figure 28 Computation Time vs Number of Iterations for Dice light field reconstruction 


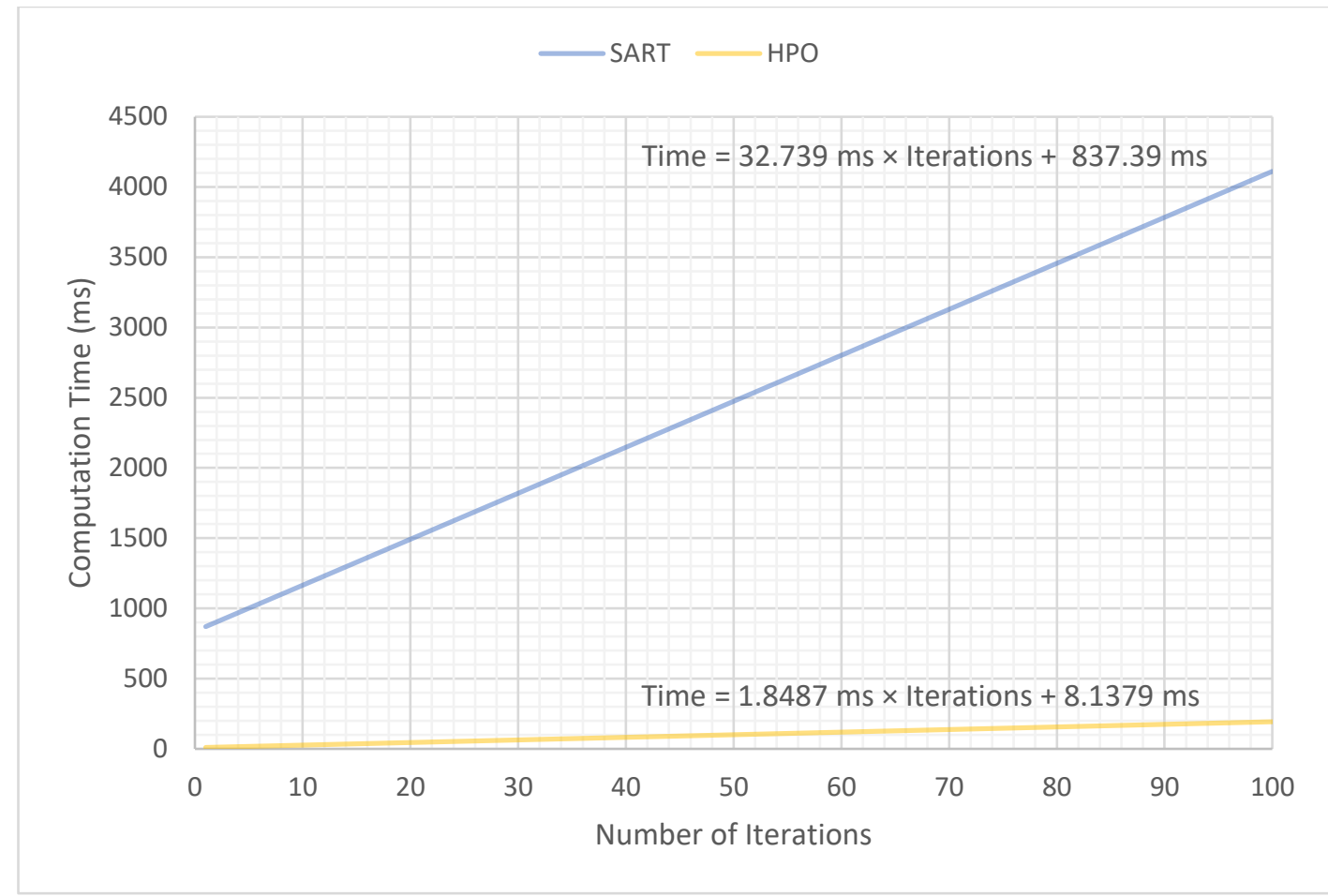

Figure 29 Computation Time vs Number of Iterations for Messerschmitt light field reconstruction

It is obvious that both algorithms' computation time grows linearly with more number of iterations. The trendlines are calculated and shown in figures, and their equations can be interpreted as a function of two variables, time and iterations, related by two factors, initialization time and iteration time.

For the Dice light field reconstruction, SART costs 832.9 milliseconds to initialize layers, while further updates each take 32.019 milliseconds on average. In contrast, HPO finish initialization in 8.0442 milliseconds and runs at 1.854 milliseconds per iteration. Similar result is obtained in Messerschmitt reconstruction.

The HPO algorithm demonstrates surprisingly high speed in computation. While its initialization time more than ten times faster than SART, it updates layers in less than 2 milliseconds. Although SART operates at about 32 milliseconds per iteration after 
initialization, it is not fast enough for real-time applications, since other operations like interaction with 3D scene will introduce more latencies and cause the overall framerate to be less than 30 frame-per-second. On the contrary, with light field decomposition costing only 2 milliseconds, sufficient time can be allocated to other operations. Current commercial displays mainly refresh at $60 \mathrm{~Hz}$, and some even can achieve $144 \mathrm{~Hz}$. With 2 milliseconds updating cycle, attractive commercial product becomes possible.

Although details of NMF algorithm regarding speed are not given, 2 milliseconds per update is still more than two times faster than the 4.44 milliseconds per iteration mentioned in the research work on NMF [12].

In addition, short initialization time of 8 milliseconds means negligible lag between scenes. Latency of $800+$ millisecond leads to visible delay when the 3D scene changes and the layers are reinitialized.

\subsection{Number of iterations vs Image quality}

Another measurement of performance of light field reconstruction is the emitted light field image's quality. One of the most common method to evaluate this is the PSNR tool, which can estimate noises inside images and has been commonly adopted for many years. Another way of assessing image quality is SSIM, which evaluate an image's distortion with an uncompressed version as reference. We applied both methods for the reconstruction of the two light fields.

Like previous time analysis, values are measured for different number of iterations from 1 to 100. 


\subsubsection{PSNR}

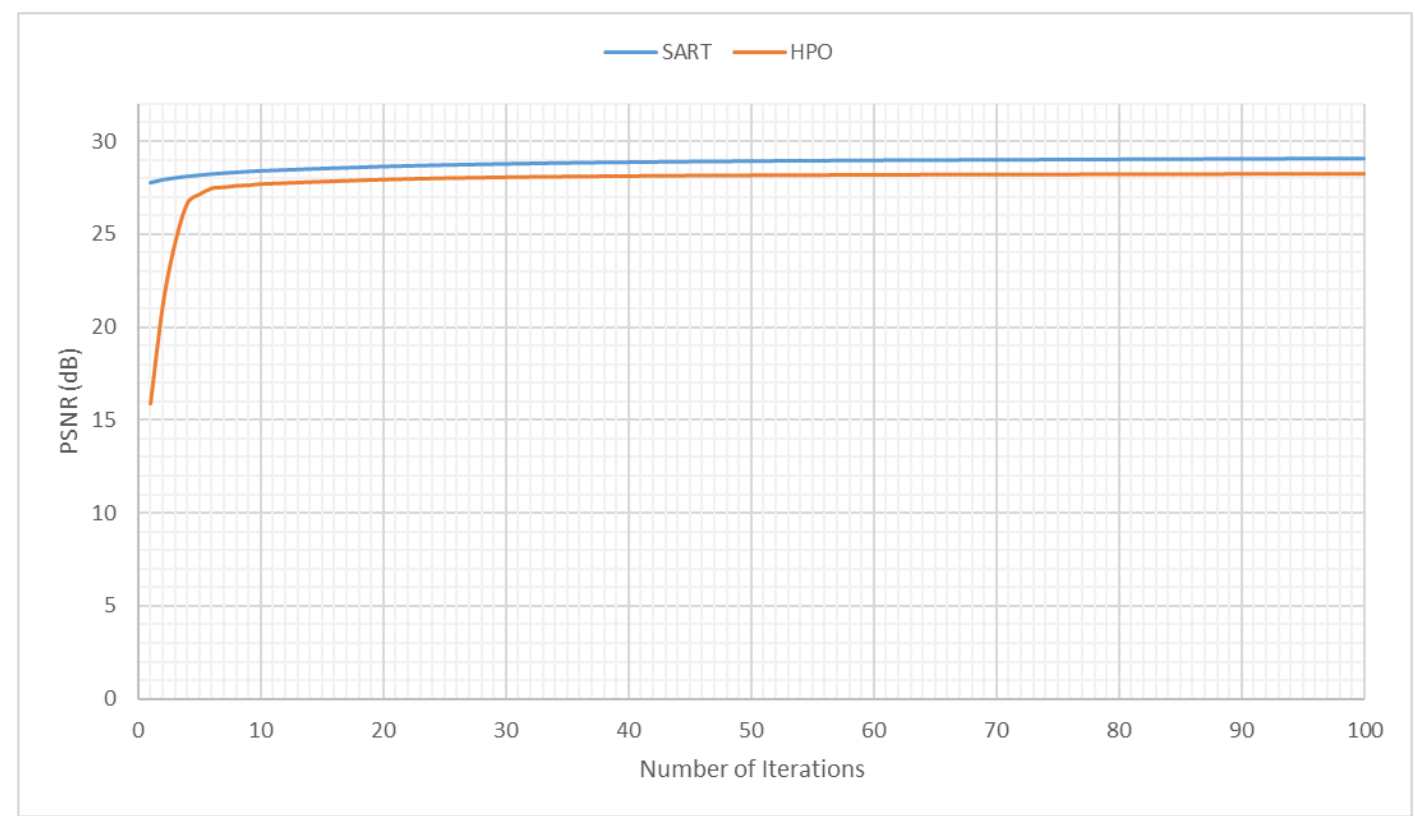

Figure 30 PSNR vs Number of Iterations for Dice light field decomposition

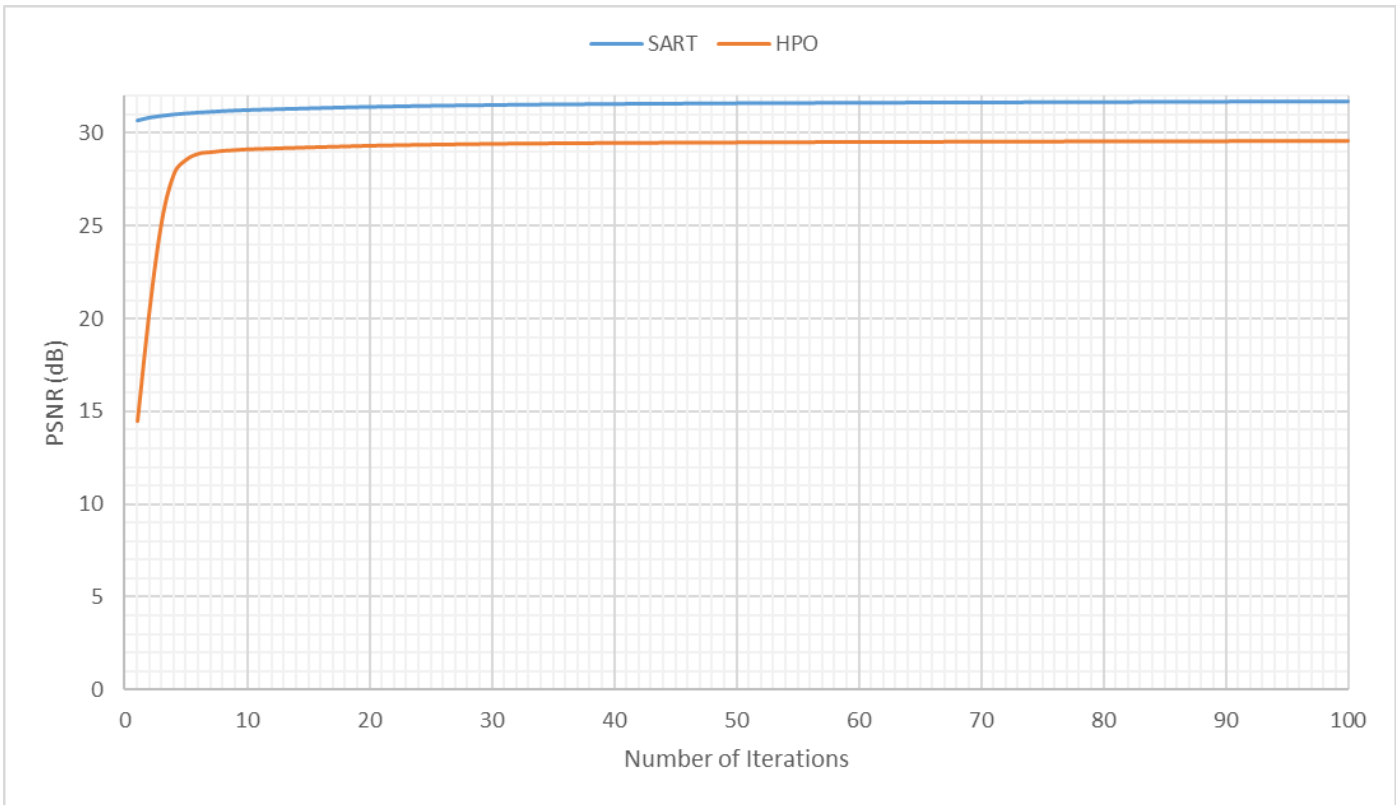

Figure 31 PSNR vs Number of Iterations for Messerschmitt light field decomposition

As shown in figures, SART can achieve high PSNR in its first iteration. This is the reason why initialization time of it is much longer than HPO. The increment of 
SART's PSNR is small afterwards. PSNR of HPO grows fast from a low value around $15 \mathrm{~dB}$ until 6 iterations, where it continues to increase at an insignificant rate. The PSNR of SART is always slightly higher than that of HPO by about $2 \mathrm{~dB}$.

Although SART reaches high PSNR in less iterations, HPO can achieve comparable PSNR around $30 \mathrm{~dB}$ in shorter time, since 6 iterations in HPO take less than 20 milliseconds while initialization of SART costs more than 800 milliseconds.

From these facts, we can say that HPO has similar image quality with SART in terms of PSNR, but HPO can achieve good image quality much faster.

\subsubsection{SSIM}

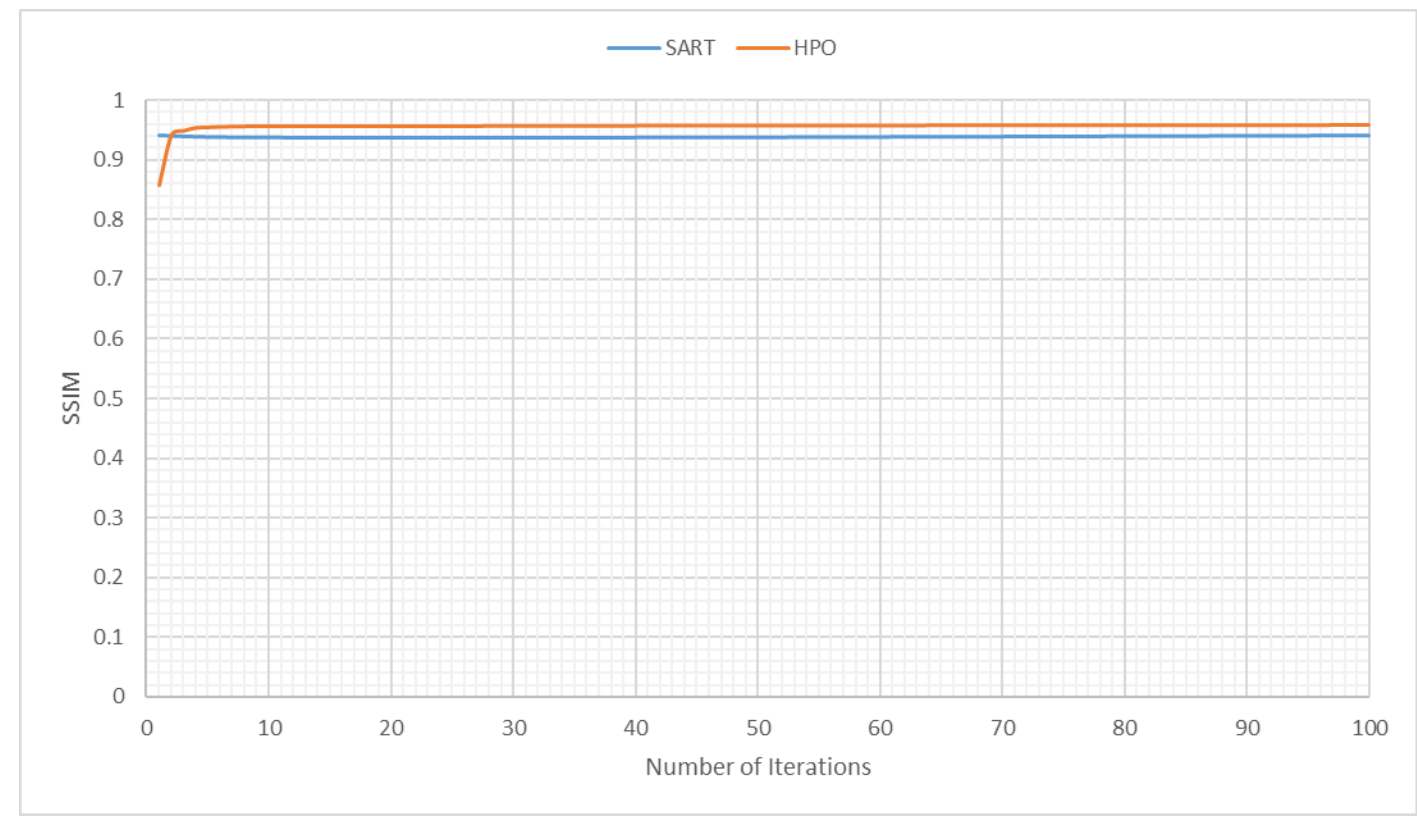

Figure 32 SSIM vs Number of Iterations for Dice light field decomposition 


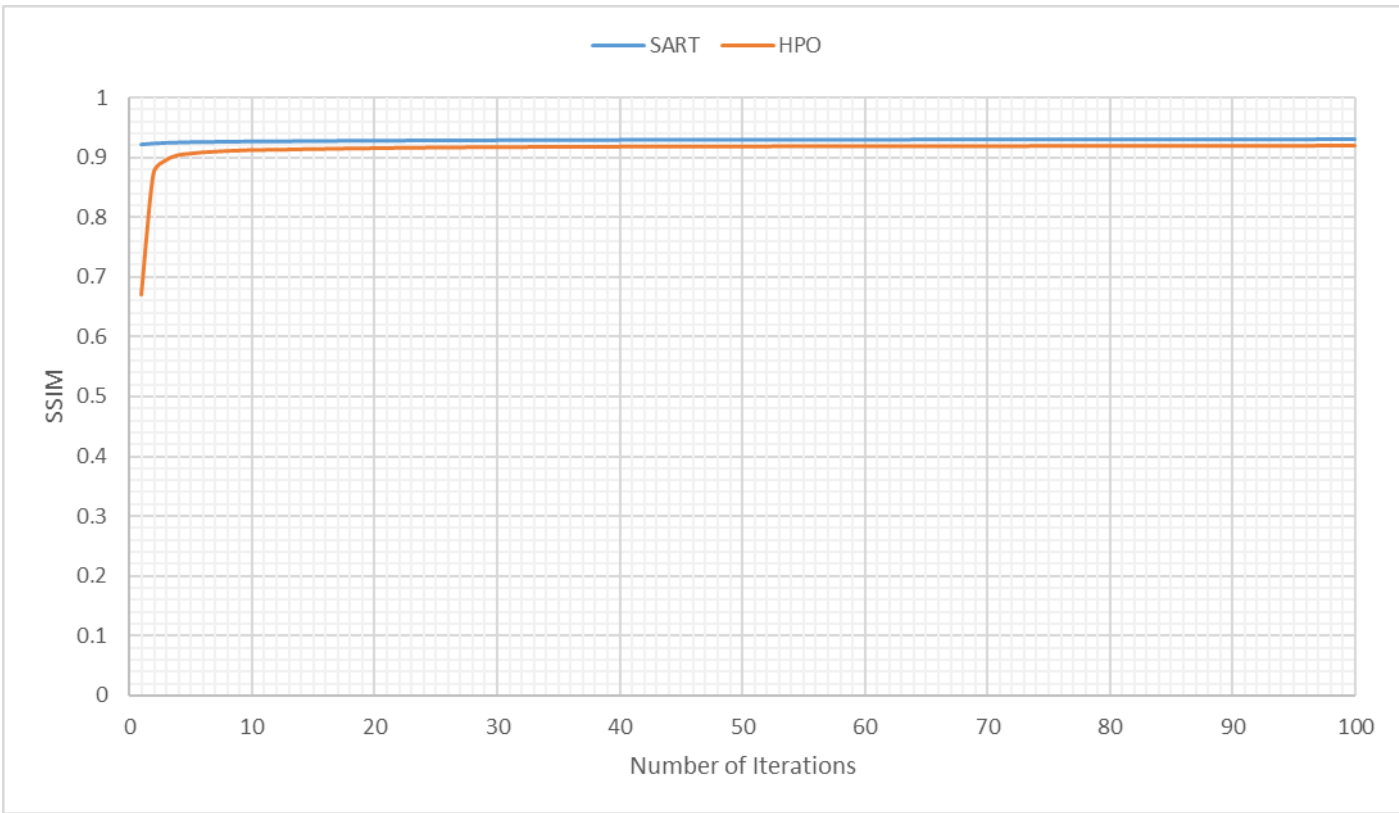

Figure 33 SSIM vs Number of Iterations for Messerschmitt light field decomposition Comparing to the PSNR assessment, SSIM is more in accordance with how human vision perceive images [21]. SSIM is ranged between 0 and 1 . The closer of the evaluated value to 1 , the higher image quality it indicates.

Similar to PSNR analysis, HPO starts with lower values first and rises quickly with more iterations until 4 iterations, then its SSIM value almost stops growing. SSIM of SART begins with high value at 1 iteration and barely increases after that.

However, in the case of Dice, the SSIM value of HPO becomes higher than that of SART. The difference after 10 iterations is about 0.02 , about two times higher than the difference in the case of Messerschmitt, where SART values are slightly higher than HPO by approximately 0.01 .

As the calculation of PSNR differs from measurement of SSIM, the difference in complexity of light field scene could have led to such reverse results. We can deduce 
that in simpler scenes like the case of Dice, HPO can achieve slightly higher image quality than SART in terms of SSIM.

If we consider the time needed to reach similar relatively high image quality, where SART uses about 830 milliseconds in 1 iteration and HPO takes about 17 milliseconds in 5 iterations, we can say that HPO achieves comparable image quality 50 times faster than SART.

In general, the overall image quality of HPO is similar to SART, while the computation speed of the former is much higher than the latter. HPO also computes faster than NMF does, as an indirect comparison indicates. 


\section{Conclusion and Recommendations}

\subsection{Conclusion}

This thesis introduced an HPO light field display with its design and algorithm to propose a light field display with greater potential as commercial product. Special backlight is adopted to support the algorithm, which split light field image set into horizontal line fractions. The image layers are calculated by pixel lines in Graphics Processing Unit (GPU) using CUDA parallel programming.

With optimizations like adjusting memory management, the HPO algorithm can decompose light fields with horizontal-only perspectives efficiently. It is proved that the algorithm can run at much higher speed than existing ones.

The new HPO light field display is capable of real-time rendering. Therefore, it is suitable for entertainment and advertising, and even more applications can be explored.

\subsection{Recommendations for further research}

There exist some limitations in this HPO work, where future researches can fill the gaps.

As demonstrated in chapter 6, two light field inputs are used for assessments between HPO and SART. More kind of inputs with various setups in 3D models and backgrounds should be tested with HPO, to find situations where the algorithm has

poor performance. Then the algorithm can be modified accordingly to achieve higher image quality regardless of input variations. 
Light fields with 7 horizontal perspectives and $10^{\circ} \mathrm{FOV}$ are examined. To increase FOV and enable more viewers, it is recommended to increase number of views. Meanwhile, since larger number of views may lead to degrade of image quality, an analysis of relationship between views, FOV, and PSNR/SSIM can be conducted to find a balance point.

Static light field inputs are evaluated for evaluation to prove benefits of this HPO algorithm, but interactive dynamic 3D scene is more attractive for real-life applications. CUDA is capable of interop with OpenGL, as it can accept light field inputs captured in real-time by OpenGL and pass resultant image layers after calculation. Viewer actions like body movement or hand gesture can be integrated to achieve interactive glasses-free 3D, therefore enhance user experience.

If this HPO light field display becomes commercialized, there will be need for fast compressing and decompressing of light field videos or streams. Using the HPO algorithm, an efficient light field video encoder/decoder can be made. 


\section{Author's Publications}

(1) S. Guo, P. Surman, Z. Zhuang and X. W. Sun, "A real-time head tracker for autostereoscopic display," in Visual Communications and Image Processing (VCIP), Singapore, 2015.

(2) Z. Zhuang, L. Zhang, P. Surman, S. Guo, B. Cao, Y. Zheng, and X. W. Sun, "Directional view method for a time-sequential autostereoscopic display with full resolution," Appl. Opt. 55, 7847-7854 (2016)

(3) S. Guo, Z. Zhuang, L. Zhang, X. Zhang, P. Surman, Y. Zheng, X. W. Sun, "Head tracked multiview display with minimum resolution loss," accepted by Photonics@SG (Paper ID: s2176)

(4) A paper on HPO hardware and software is planned to be submitted to Optics Express soon. 


\section{Bibliography}

[1] F. E. Ives, "A novel stereogram," Journal of the Franklin Institute, pp. 51-52, 1902.

[2] T. L. N. E. a. S. B. Adrian Travis, "Collimated light from a waveguide for a display backlight," Optics Express, vol. 17, no. 22, pp. 19714-19719, 2009.

[3] G. Lippmann, "Epreuves Reversibles Donnant la Sensation du Relief," Journal of Theoretical and Applied Physics, vol. 7, no. 1, pp. 821-825, 1908.

[4] D. L. MacFarlane, "Volumetric three-dimensional display," Applied Optics, vol. 33, no. 31, pp. 7453-7457, 1994.

[5] C. C. M. S. Chris Slinger, "Computer-generated holography as a generic display technology," Computer, vol. 38, no. 8, pp. 46-53, 2005.

[6] P. H. Marc Levoy, "Light field rendering," Proceedings of the 23rd annual conference on Computer graphics and interactive techniques, pp. 31-42, 1996.

[7] M. M. Lab, "Synthetic Light Field Archive," MIT Media Lab, [Online]. Available: $\quad$ http://web.media.mit.edu/ gordonw/SyntheticLightFields/. [Accessed 2017].

[8] D. L. W. H. R. R. Gordon Wetzstein, "Layered 3D: tomographic image synthesis for attenuation-based light field and high dynamic range displays," 
ACM Transactions on Graphics (TOG) - Proceedings of ACM SIGGRAPH 2011, vol. 30, no. 4, 2011.

[9] M. H. Y. K. R. R. Douglas Lanman, "Content-Adaptive Parallax Barriers: Optimizing Dual-Layer 3D Displays using Low-Rank Light Field Factorization," ACM Transactions on Graphics (TOG) - Proceedings of ACM SIGGRAPH Asia 2010, vol. 29, no. 6, 2010.

[10] D. L. M. H. R. R. Gordon Wetzstein, "Tensor displays: compressive light field synthesis using multilayer displays with directional backlighting," $A C M$ Transactions on Graphics (TOG) - Proceedings of ACM SIGGRAPH 2012, vol. 31, no. 4, 2012.

[11] G. W. M. H. W. H. R. R. Douglas Lanman, "Polarization Fields: Dynamic Light Field Display using Multi-Layer LCDs," ACM Transactions on Graphics (TOG) - Proceedings of ACM SIGGRAPH Asia 2011, vol. 30, no. 6, 2011.

[12] R. C. H. F. R. R. G. W. Andrew Maimone, "Wide field of view compressive light field display using a multilayer architecture and tracked viewers," Journal of the Society for Information Display, 2015.

[13] Z. G. M. Z. X. Z. Xuan Cao, "Load-Balancing Multi-LCD Light Field Display," Stereoscopic Displays and Applications Conference Proceedings, 2015. 
[14] C. J. S. M. B. L. B. L. Seungjae Lee, "Additive light field displays: realization of augmented reality with holographic optical elements," ACM Transactions on Graphics, vol. 35, no. 4, pp. 1-13, 2016.

[15] K. C. G. W. Fu-Chung Huang, "The light field stereoscope: immersive computer graphics via factored near-eye light field displays with focus cues," ACM Transactions on Graphics (TOG) - Proceedings of ACM SIGGRAPH 2015, vol. 34, no. 4, 2015.

[16] B. R. Noel Lopes, "Non-negative Matrix Factorization Implementation Using Graphic Processing Units," International Conference on Intelligent Data Engineering and Automated Learning, pp. 275-283, 2010.

[17] X. S. X. Y. X. Z. S. X. N. G. Duo Chen, "Performance improvement of compressive light field display with the viewing-position-dependent," Optics Express, vol. 24, no. 26, 2016.

[18] Z. G. T. L. M. Z. a. Z. Z. Xuan Cao, "Accelerating decomposition of light field video for compressive multi-layer display.," Optics Express, vol. 23, no. 26, 2015.

[19] NVidia, "CUDA Parallel Computing Platform," NVidia, 2017. [Online]. Available: http://www.nvidia.com/object/cuda_home_new.html. [Accessed $2017]$.

[20] A. Weber, "The USC-SIPI Image Database," The USC-SIPI, 1981. [Online]. Available: http://sipi.usc.edu/database/. [Accessed 2017]. 
[21] A. B. H. S. E. S. Zhou Wang, "Image quality assessment: from error visibility to structural similarity," IEEE Transactions on Image Processing, vol. 13, no. 4, pp. 600-612, 2004. 